\title{
On Classification of Finite-Dimensional Superbialgebras and Hopf Superalgebras
}

\author{
Said AISSAOUI ${ }^{\dagger}$ and Abdenacer MAKHLOUF ${ }^{\ddagger}$ \\ † Université A-Mira, Laboratoire de Mathématiques Appliquées, \\ Targa Ouzemmour 06000 Béjaia, Algeria \\ E-mail: aissaoui.said@yahoo.fr \\ ‡Université de Haute Alsace, Laboratoire de Mathématiques, Informatique et Applications, \\ 4, rue des Frères Lumière F-68093 Mulhouse, France \\ E-mail: Abdenacer.Makhlouf@uha.fr \\ URL: http://www.algebre.fst.uha.fr/makhlouf/Makhlouf .html
}

Received February 08, 2013, in final form December 23, 2013; Published online January 02, 2014 http://dx.doi.org/10.3842/SIGMA.2014.001

\begin{abstract}
The purpose of this paper is to investigate finite-dimensional superbialgebras and Hopf superalgebras. We study connected superbialgebras and provide a classification of non-trivial superbialgebras and Hopf superalgebras in dimension $n$ with $n \leq 4$.
\end{abstract}

Key words: superalgebra; superbialgebra; Hopf superalgebra; classification

2010 Mathematics Subject Classification: 16T10; 57T05; 17A70

\section{Introduction}

Hopf superalgebras are a generalization of the supergroup notion. In physics, infinite-dimensional Hopf superalgebras based on Lie superalgebras turn out to be related to the integrable $S$-matrix of the AdS/CFT correspondence. For a large class of finite-dimensional Lie superalgebras (including the classical simple ones) a Lie supergroup associated to the algebra is defined by fixing the Hopf superalgebra of functions on the supergroup [34]. This framework seems to be interesting for studying supermanifolds and supersymmetry. Majid showed that the theory of Lie superalgebras and Hopf superalgebras can be reduced to the classical case using the bosonization by $\mathbb{K}[\mathbb{Z} / 2 \mathbb{Z}]$, where $\mathbb{K}$ is an algebraically closed field [24]. Recently some classes of Hopf superalgebras were investigated like for example pointed Hopf superalgebras and quasitriangular Hopf superalgebras [3, 4, 18], see also [13, 23]. So far very few is known about general classification of superbialgebras and Hopf superalgebras. Nevertheless, classification of finite-dimensional Hopf algebras is known for small dimensions and structure of many classes are deeply studied, see for example $[2,4,5,9,10,15,16,28,29,31,32,33,36,37,38$, 39]. For general theory of Hopf algebras, we refer to [1, 19, 21, 25, 30, 35], and for applications see for example [12, 20, 22, 27].

In this paper, we discuss properties of $n$-dimensional superbialgebras and provide a classification of non-trivial superbialgebras in dimensions 2, 3 and 4. Moreover we derive a classification of Hopf superalgebras for these dimensions.

The paper is organized as follows. Section 1 reviews definitions and properties of superbialgebras and Hopf superalgebras. Moreover, we provide an outline of the computations leading to classifications. In Section 2, we focus on trivial and connected superbialgebras. We characterize connected superbialgebras and classify connected 2-dimensional superbialgebras and Hopf superalgebras. Sections 3 and 4 deal respectively with classification of 3 - and 4 -dimensional superbialgebras and Hopf superalgebras. We emphasize on non-trivial superbialgebras, from 
which we derive the non-trivial Hopf superalgebras. To this end, we establish classification of 3-dimensional superalgebras and use the 4-dimensional classification due to Armour, Chen and Zhang [7].

Throughout this paper, we work over $\mathbb{K}$, an algebraically closed field of characteristic zero. Unless otherwise specified, we will denote the multiplication by $\mu$ or $\cdot$, and the unit element by $\eta(1)=e_{1}=e_{1}^{0}=1$. For simplicity, we will use just product (or multiplication), unit, counit and coproduct (or comultiplication) in the sense of even morphism (i.e. we will drop the suffix 'super'). We also would like to mention that in the last section, most of the results are obtained by using the computer algebra system Mathematica. The program we have used is available.

\section{Definitions of superbialgebras and Hopf superalgebras}

In this section, we summarize definitions and properties of superbialgebras and Hopf superalgebras. For more details, we refer to [3, 18].

A superspace $A$ is a $\mathbb{K}$-vector space endowed with a $\mathbb{Z} / 2 \mathbb{Z}$-grading, in other words, it writes as a direct sum of two vector spaces $A=A_{0} \oplus A_{1}$ such as $A_{0}$ is the even part and $A_{1}$ is the odd part. Elements of $A_{0}$ (resp. $A_{1}$ ) are called even homogeneous (resp. odd homogeneous). If $a \in A_{0}$, we set $|a|=\operatorname{deg}(a)=0$ and if $a \in A_{1},|a|=\operatorname{deg}(a)=1$. Notice that some authors denote the dimension of $A$ by $i \mid j$, such that $i=n_{0}$ and $j=n_{1}$, where $n_{0}=\operatorname{dim} A_{0}$ and $n_{1}=\operatorname{dim} A_{1}$. But in this paper we preserve this notation for the superalgebra obtained by the $i$ th algebra. Notice that the following definitions could be described in a symmetric category of superspaces.

Definition 1.1. A superalgebra is a triple $(A, \mu, \eta)$ where $A$ is a superspace, $\mu: A \otimes A \rightarrow A$ (multiplication) and $\eta: \mathbb{K} \rightarrow A$ (unit) are two superspace morphisms satisfying

$$
\begin{aligned}
& \mu \circ\left(\mu \otimes \operatorname{id}_{A}\right)=\mu \circ\left(\operatorname{id}_{A} \otimes \mu\right) \quad \text { (associativity), } \\
& \mu \circ \eta \otimes \operatorname{id}_{A}=\mu \circ \operatorname{id}_{A} \otimes \eta \quad \text { (unity). }
\end{aligned}
$$

A superalgebra $A$ is called commutative if the multiplication satisfies $\mu \circ \tau=\mu$ where $\tau$ is a superflip. In other words, for all homogeneous elements $a, b \in A, \mu(a \otimes b)=(-1)^{|a||b|} \mu(b \otimes a)$.

Let $\left(A, \mu_{A}, \eta_{A}\right),\left(B, \mu_{B}, \eta_{B}\right)$ be two superalgebras. A map $f: A \rightarrow B$ is a superalgebra morphism if it is a superspace morphism satisfying

$$
f \circ \mu_{A}=\mu_{B} \circ f \otimes f \quad \text { and } \quad f \circ \eta_{A}=\eta_{B} .
$$

Definition 1.2. A supercoalgebra is a triple $(C, \Delta, \varepsilon)$ where $C$ is a superspace such that $\Delta: C \rightarrow$ $C \otimes C$ (comultiplication or coproduct) and $\varepsilon: C \rightarrow \mathbb{K}$ (counit) are two superspace morphisms satisfying

$$
\begin{aligned}
& \left(\Delta \otimes \operatorname{id}_{C}\right) \circ \Delta=\left(\operatorname{id}_{C} \otimes \Delta\right) \circ \Delta \quad \text { (coassociativity) } \\
& \left(\varepsilon \otimes \operatorname{id}_{C}\right) \circ \Delta=\left(\operatorname{id}_{C} \otimes \varepsilon\right) \circ \Delta \quad \text { (counity). }
\end{aligned}
$$

We use the Sweedleer's notation for the coproduct, we set for all $x \in C$

$$
\Delta(x)=\sum_{(x)} x^{(1)} \otimes x^{(2)}, \quad(\mathrm{id} \otimes \Delta) \circ \Delta(x)=(\Delta \otimes \mathrm{id}) \circ \Delta(x)=\sum_{(x)} x^{(1)} \otimes x^{(2)} \otimes x^{(3)} .
$$

A supercoalgebra $C$ is said to be cocommutative if the comultiplication satisfies $\Delta=\tau \circ \Delta$, where $\tau$ is the superflip, that is

$$
\forall x \in C, \quad \Delta(x)=\sum_{(x)} x^{(1)} \otimes x^{(2)}=\sum_{(x)}(-1)^{\left|x^{(1)}\right|\left|x^{(2)}\right|} x^{(2)} \otimes x^{(1)} .
$$


Let $\left(A, \Delta_{A}, \varepsilon_{A}\right),\left(B, \Delta_{B}, \varepsilon_{B}\right)$ be two supercoalgebras. The map $f:\left(A, \Delta_{A}, \varepsilon_{A}\right) \rightarrow\left(B, \Delta_{B}, \varepsilon_{B}\right)$ is a supercoalgebra morphism if it is a superspace morphism and satisfies

$$
f \otimes f \circ \Delta_{A}=\Delta_{B} \circ f \quad \text { and } \quad \varepsilon_{B} \circ f=\varepsilon_{A} .
$$

Now, we consider a superbialgebra structure which is obtained by combining superalgebras and supercoalgebras. Moreover, if a superbialgebra has an anti-superbialgebra morphism satisfying some condition, it is called a Hopf superalgebra.

Definition 1.3. A superbialgebra is a tuple $(A, \mu, \eta, \Delta, \varepsilon)$, where $(A, \mu, \eta)$ is a superalgebra and $(A, \Delta, \varepsilon)$ is a supercoalgebra such that one of these two equivalent compatibility conditions hold:

1) $\Delta: A \rightarrow A \otimes A$ and $\varepsilon: A \rightarrow \mathbb{K}$ are superalgebra morphisms,

2) $\mu: A \otimes A \rightarrow A$ and $\eta: \mathbb{K} \rightarrow A$ are supercoalgebra morphisms.

In other words, $\Delta$ (resp. $\varepsilon$ ) satisfies the following compatibility condition

$$
\begin{aligned}
& \Delta \circ \mu=(\mu \otimes \mu) \circ\left(\operatorname{id}_{A} \otimes \tau \otimes \operatorname{id}_{A}\right) \circ(\Delta \otimes \Delta), \quad \Delta \circ \eta=\eta \otimes \eta \\
& \left(\text { resp. } \quad \varepsilon \circ \mu=\mu_{\mathbb{K}} \circ(\varepsilon \otimes \varepsilon), \quad \varepsilon \circ \eta=\operatorname{id}_{\mathbb{K}}\right),
\end{aligned}
$$

where $\mu_{\mathbb{K}}$ is the multiplication of $\mathbb{K}$.

The last condition in (1.7) says that the unit $1=e_{1}^{0}=\eta(1)$ of the superbialgebra is a grouplike element $\Delta(1)=1 \otimes 1$.

Remark 1.4. Note that if $\left(A=A_{0} \oplus A_{1}, \mu, \eta, \Delta, \varepsilon\right)$ is a superbialgebra with $\eta(1)=1$, a unit element, then we have

$$
\varepsilon\left(A_{1}\right)=0 \quad \text { and } \quad \varepsilon(1)=1 .
$$

Indeed, if $\varepsilon: A \longrightarrow \mathbb{K}$ is a superspace morphism and $\mathbb{K}$ is a superspace whose odd part is 0 , then $\varepsilon$ sends the odd part of $\mathrm{A}$ to odd part of $\mathbb{K}$ which is 0 . For the second assertion, if $A$ is a superbialgebra then $\varepsilon$ is a superalgebra morphism and it sends the unit element of $A$ to unit element of $\mathbb{K}$.

If $A$ and $B$ are two superbialgebras over $\mathbb{K}$, we shall call a superspace morphism $f: A \rightarrow B$ a superbialgebra morphism if it is both a superalgebra morphism and a supercoalgebra morphism.

Definition 1.5. A Hopf superalgebra is a superbialgebra admitting an antipode, that is a superspace morphism $S: A \rightarrow A$ which satisfies the following condition:

$$
\mu \circ\left(S \otimes \operatorname{id}_{A}\right) \circ \Delta=\mu \circ\left(\operatorname{id}_{A} \otimes S\right) \circ \Delta=\eta \circ \varepsilon .
$$

A Hopf superalgebra is given by a tuple $H=(A, \mu, \eta, \Delta, \varepsilon, S)$.

Let $(A, \mu, \eta, \Delta, \varepsilon, S)$ be a Hopf superalgebra and $S$ its antipode. Then we have the following properties:

1) $S \circ \mu=\mu \circ \tau \circ(S \otimes S)$;

2) $S \circ \eta=\eta$;

3) $\varepsilon \circ S=\varepsilon$;

4) $\tau \circ(S \otimes S) \circ \Delta=\Delta \circ S$;

5) if $A$ is commutative or cocommutative then $S \circ S=$ id where id :A $A A$ is the identity morphism; 
6 ) if the antipode $S$ of the Hopf superalgebra $A$, with $\Delta$ as comultiplication, is bijective then $A$ is also a Hopf superalgebra, with the opposite comultiplication $\Delta^{\prime}=\tau \circ \Delta$ and the opposite antipode $S^{\prime}=S^{-1}$;

7) all finite-dimensional Hopf superalgebras possess bijective antipodes.

Remark 1.6. Some superalgebras do not carry a superbialgebra structure. For example $A=$ $M_{2}(\mathbb{K})$ is a superalgebra with matrix multiplication and such that the even part is $A_{0}=$ $\mathbb{K} e_{1}^{0} \oplus \mathbb{K} e_{2}^{0}$ and the odd part is $A_{1}=\mathbb{K} e_{1}^{1} \oplus \mathbb{K} e_{2}^{1}$, where

$$
e_{1}^{0}=\left(\begin{array}{ll}
1 & 0 \\
0 & 1
\end{array}\right), \quad e_{2}^{0}=\left(\begin{array}{ll}
1 & 0 \\
0 & 0
\end{array}\right), \quad e_{1}^{1}=\left(\begin{array}{ll}
0 & 1 \\
0 & 0
\end{array}\right), \quad e_{2}^{1}=\left(\begin{array}{ll}
0 & 0 \\
1 & 0
\end{array}\right) .
$$

Indeed, the compatibility condition between the counit $\varepsilon$ and the product $\varepsilon(\mu(x \otimes y))=\varepsilon(x) \varepsilon(y)$ isn't always satisfied, since in one hand we have $\varepsilon\left(\mu\left(e_{1}^{1} \otimes e_{2}^{1}\right)\right)=\varepsilon\left(e_{2}^{0}\right)=0$, because $\varepsilon\left(e_{2}^{1}\right)=$ $\varepsilon\left(e_{1}^{1}\right)=0$, and in the other hand $\varepsilon\left(\mu\left(e_{2}^{1} \otimes e_{1}^{1}\right)\right)=\varepsilon\left(e_{1}^{0}-e_{2}^{0}\right)=0$. Then $\varepsilon\left(e_{2}^{0}\right)=1$, which leads to a contradiction.

In Sections 3 and 4, we aim to classify $n$-dimensional non-trivial superbialgebras (resp. Hopf superalgebras), for $n=3$ and $n=4$. An $n$-dimensional superbialgebra (resp. a Hopf superalgebra) is identified to its structure constants with respect to a fixed basis. It turns out that the axioms of superbialgebra structure translate to a system of polynomial equations that define the algebraic variety of $n$-dimensional superbialgebras which is embedded into $\mathbb{K}^{2 n_{0}^{3}+6 n_{0} n_{1}^{2}+n_{0}-1}$. The classification requires to solve this algebraic system. The calculations are handled using a computer algebra system. We include in the following an outline of the computation.

1. We provide a list of non-trivial $n$-dimensional superalgebras:

- For $n=2$, the list is taken from $[14,17]$.

- For $n=3$, the list is given in Propositions 3.2 and 3.3.

- For $n=4$, the list is taken from [6].

2. Fix a superalgebra $A$ and compute the supercoalgebra's structure constants $\left\{D_{(i, s),(j, t)}^{k}\right\}$ and $\left\{\xi_{i}^{0}\right\}$, where

$$
\Delta\left(e_{i}^{l}\right)=\sum_{s=0}^{1} \sum_{j=1}^{n_{s}} \sum_{k=1}^{n_{t}} D_{(i, l)}^{(j, s)(k)} e_{j}^{s} \otimes e_{k}^{t}
$$

with $t=(l+s) \bmod [2]$ and

$$
\varepsilon\left(e_{i}^{l}\right)= \begin{cases}\xi_{i}^{0} & \text { if } \quad l=0 \text { and } i \neq 1, \\ 0 & \text { if } \quad l=1, \\ 1 & \text { if } \quad l=0 \text { and } i=1\end{cases}
$$

with respect to bases $\left\{e_{i}^{0}\right\}_{i=1, \ldots, n_{0}}$ and $\left\{e_{i}^{1}\right\}_{i=1, \ldots, n_{1}}$ of $A_{0}$ and $A_{1}$, respectively. Supercoalgebra structures on $A$ making it a superbialgebra are in one-to-one correspondence with solutions of the system corresponding to (1.4), (1.5), (1.7).

3. Obtained in (2) is a family $\left\{A_{i}\right\}_{i \in I}$ of superalgebras whose underlying superalgebra is $A$. We sort them into isomorphism classes. Two superbialgebras, given by their structure constants, are isomorphic if there exist matrices $\left(T_{(i, 0)}^{k}, T_{(i, 1)}^{k}\right)_{i, k}$ defining a superbialgebra morphism with respect to the basis, that is satisfying $\operatorname{det}\left(T_{(i, 0)}^{k}\right) \operatorname{det}\left(T_{(i, 1)}^{k}\right) \neq 0$ and (1.3)-(1.6).

4. For each representative of isomorphism classes, determine whether it admits an antipode or not. It follows from existence or not of structure constants $\lambda_{(i, s)}^{k}$, of a morphism $S$, $S\left(e_{i}^{s}\right)=\sum_{k=1}^{n_{s}} \lambda_{(i, s)}^{k} e_{k}^{s}$, satisfying (1.8). 


\section{Trivial and connected superbialgebras}

We describe in this section some properties of connected superbialgebras, which are superbialgebras with 1-dimensional even part and trivial superbialgebras, which are superbialgebras with trivial odd part.

\subsection{Trivial superbialgebras}

We have the following obvious result.

Proposition 2.1. Every finite-dimensional bialgebra (resp. Hopf algebra) is a superbialgebra (resp. Hopf superalgebra), whose odd part is reduced to $\{0\}$. This superbialgebra (resp. Hopf superalgebra) is called trivial superbialgebra (resp. trivial Hopf superalgebra).

The algebraic classification of Hopf algebra were investigated by many authors. Hopf algebras of prime dimensions were classified in [39]. We refer to [37, 38] for the classification of finitedimensional Hopf algebras up to dimension 11, see also [26]. Semisimple Hopf algebras of dimension 12 were given in [16] and complete classification of dimension 12 provided in [32]. See [8] for dimension 14 and [11] for dimension 20. The minimal dimension where the problem is unsolved is 24. For the classification of 2 and 3-dimensional bialgebras, we refer to [14]. They are based on Gabriel's result of 2 and 3-dimensional algebras [17]. Classification of 4-dimensional superbialgebras will be given in a forthcoming paper.

\subsection{Connected superbialgebras}

In the following we consider connected superbialgebras whose even part are isomorphic to $\mathbb{K}$. The notions of a connected superalgebra, supercoalgebra and Hopf superalgebra are defined in the same way.

Lemma 2.2. Let $A=A_{0} \oplus A_{1}$ be an $(n+1)$-dimensional connected superspace. If $(A, \mu, \eta, \Delta, \varepsilon)$ is a superbialgebra with $1=\eta(1)$, then we have

1) $\mu(x \otimes y)=\mu(y \otimes x)=0, \forall x, y \in A_{1}$,

2) $\Delta(x)=1 \otimes x+x \otimes 1, \forall x \in A_{1}$.

Proof. Let $\left(x_{1}, x_{2}, \ldots, x_{n}\right)$ be a basis of $A_{1}$, and set $\mu\left(x_{i} \otimes x_{j}\right)=\alpha_{i j} 1, \Delta\left(x_{i}\right)=\sum_{k=1}^{n}\left(\lambda_{k 1} x_{k} \otimes\right.$ $\left.1+\lambda_{1 k} 1 \otimes x_{k}\right)$, with $\alpha_{i j}, \lambda_{1 j}, \lambda_{j 1} \in \mathbb{K}, \forall i, j \in\{1, \ldots, n\}$.

- The compatibility of the counit and the multiplication $\varepsilon\left(\mu\left(x_{i} \otimes x_{j}\right)\right)=\varepsilon\left(x_{i}\right) \varepsilon\left(x_{j}\right)$ and the fact that $\varepsilon\left(x_{i}\right)=0, \forall i \in\{1, \ldots, n\}$, show that $\alpha_{i j}=0, \forall i, j \in\{1, \ldots, n\}$. Then we have $\mu\left(x_{i} \otimes x_{j}\right)=\mu\left(x_{j} \otimes x_{i}\right)=0, \forall i, j \in\{1, \ldots, n\}$.

- The compatibility of the counit and the following condition:

$$
(\varepsilon \otimes \mathrm{id})\left(\Delta\left(x_{i}\right)\right)=(\operatorname{id} \otimes \varepsilon)\left(\Delta\left(x_{i}\right)\right)=x_{i}, \quad \forall i \in\{1, \ldots, n\},
$$

lead to $(\varepsilon \otimes \mathrm{id})\left(\Delta\left(x_{i}\right)\right)=\sum_{k=1}^{n}\left(\lambda_{k 1} \varepsilon\left(x_{k}\right) \otimes 1+\lambda_{1 k} \varepsilon(1) \otimes x_{k}\right)=\sum_{k=1}^{n} \lambda_{1 k} x_{k}=x_{i}$ and

$$
(\mathrm{id} \otimes \varepsilon)\left(\Delta\left(x_{i}\right)\right)=\sum_{k=1}^{n}\left(\lambda_{k 1} x_{k} \otimes \varepsilon(1)+\lambda_{1 k} 1 \otimes \varepsilon\left(x_{k}\right)\right)=\sum_{k=1}^{n} \lambda_{k 1} x_{k}=x_{i} .
$$

Then $\lambda_{1 k}=\lambda_{k 1}=0, \forall k \in\{1, \ldots, n\} \backslash\{i\}$ and $\lambda_{1 i}=\lambda_{i 1}=1$. So

$$
\Delta\left(x_{i}\right)=x_{i} \otimes 1+1 \otimes x_{i}, \quad \forall i \in\{1, \ldots, n\} .
$$


Notice that the first assertion of this lemma is just Proposition 2.11 in [6] for which the proof is different.

Theorem 2.3. An n-dimensional connected superbialgebra exists only when $n<3$.

Proof. Let $A=A_{0} \oplus A_{1}$ be a superalgebra with multiplication $\mu$ and unit 1 . Assume $A_{0}=\mathbb{K} 1$, $\operatorname{dim} A_{1}=n-1$ and $\left(x_{1}, x_{2}, \ldots, x_{n-1}\right)$ be a basis of $A_{1}$.

According to Lemma 2.2, $\Delta\left(x_{i}\right)=1 \otimes x_{i}+x_{i} \otimes 1$. Compatibility condition leads in one hand to $\Delta\left(\mu\left(x_{i} \otimes x_{j}\right)\right)=0$, and in the other hand to

$$
\begin{aligned}
& \mu \otimes \mu \circ \tau\left(\Delta\left(x_{i}\right) \otimes \Delta\left(x_{j}\right)\right)=\mu \otimes \mu \circ \tau\left(\left(1 \otimes x_{i}+x_{i} \otimes 1\right) \otimes\left(1 \otimes x_{j}+x_{j} \otimes 1\right)\right) \\
& \quad=\mu \otimes \mu \circ \tau\left(x_{i} \otimes 1 \otimes x_{j} \otimes 1+x_{i} \otimes 1 \otimes 1 \otimes x_{j}+1 \otimes x_{i} \otimes x_{j} \otimes 1+1 \otimes x_{i} \otimes 1 \otimes x_{j}\right) \\
& \quad=x_{i} \otimes x_{j}-x_{j} \otimes x_{i}, \quad \forall i, j \in\{1, \ldots, n-1\} .
\end{aligned}
$$

Since $x_{i} \otimes x_{j}-x_{j} \otimes x_{i} \neq 0, \forall i \neq j$, therefore, the compatibility condition is satisfied only if $n=2$.

A 2-dimensional superbialgebra is either trivial or connected. For trivial 2-dimensional bialgebras, we refer to [14]. In the following, we provide connected 2-dimensional superbialgebras and Hopf superalgebras classification.

Let $(A, \mu, \eta, \Delta, \varepsilon)$ be a 2 -dimensional connected superbialgebra. We set $A_{0}=\mathbb{K}, A_{1}=$ $\operatorname{span}\{x\}$ and $\eta(1)=1$ its unit element.

Proposition 2.4. Every 2-dimensional connected superbialgebra is isomorphic to 2-dimensional connected superbialgebra $\mathbb{K}[x] /\left(x^{2}\right)$ with $\operatorname{deg}(x)=1$ and defined by $\Delta(x)=1 \otimes x+x \otimes 1$.

Moreover, it carries a Hopf superalgebra structure with an antipode $S$ defined by $S(1)=1$ and $S(x)=-x$.

Proof. The multiplication, the comultiplication and the counit are defined according to Lemma 2.2 and with this extra product, coproduct and counit $x \cdot x=\alpha 1, \Delta(x)=\beta 1 \otimes x+\gamma x \otimes 1$, where $\alpha, \beta, \gamma \in \mathbb{K}$. Solving the system corresponding to conditions (1.1), (1.2), with respect to structure constants $\alpha, \beta, \gamma$, leads to the result. For the second assertion, we assume that the antipode $S$ is defined as, $S(1)=1, S(x)=\lambda x$, with $\lambda \in \mathbb{K}$. Applying the identity (1.8) to $x$, we obtain only one non trivial 2-dimensional Hopf superalgebra associated to connected 2dimensional superbialgebra defined above. The antipode is defined as $S(1)=1, S(x)=-x$.

Let $f: A \longrightarrow A$ be a superalgebra homomorphism, this means that $f$ is an even linear map, satisfying $f(1)=1$ and $f \circ \mu=\mu \circ f \otimes f$. Let us set $f(x)=\alpha x$, where $\alpha \in \mathbb{K} \backslash\{0\}$. A direct calculation shows that $f \circ \mu=\mu \circ f \otimes f$ is satisfied for any $\alpha \neq 0$. Therefore, the automorphism group of the 2-dimensional superbialgebra is the infinite group

$$
\left\{\left(\begin{array}{ll}
1 & 0 \\
0 & \alpha
\end{array}\right), \text { with } \alpha \in \mathbb{K} \backslash\{0\}\right\} \text {. }
$$

\section{Classification of 3-dimensional superbialgebras and Hopf superalgebras}

In dimension 3 , there are three cases for $n_{0}=\operatorname{dim} A_{0}$. If $n_{0}=1$, we have connected superbialgebras which are covered by Proposition 2.3. If $n_{0}=3$, we have trivial superbialgebras for which we refer to [14] for the classification of 3-dimensional bialgebras. It remains to study the case $n_{0}=2$. 


\subsection{Superalgebras}

Let $(A, \mu, \eta, \Delta, \varepsilon)$ be a 3 -dimensional superbialgebra such that $A=A_{0} \oplus A_{1}$ and $\operatorname{dim} A_{0}=2$. Let $\{1, x, y\}$ be a basis of $A$, such that $\{1, x\}$ generates the even part $A_{0}$ and $\{y\}$ generates the odd part $A_{1}$. Assume that $\eta(1)=1$.

We recall here the classification of 2-dimensional algebras.

Proposition 3.1 ([14, 17]). There are, up to isomorphism, two 2-dimensional algebras $A_{1}=$ $\mathbb{K}[x] /\left(x^{2}\right)$ and $A_{2}=\mathbb{K}[x] /\left(x^{2}-x\right)$.

Since the even part of a superalgebra is an algebra, we fix the even part multiplication to be one of the two 2-dimensional algebras, recalled in Proposition 3.1. We denote by $\mu_{i \mid j}$ the $j^{\text {th }}$ multiplication obtained by extending the multiplication $\mu_{i}$ of a 2-dimensional algebra to the multiplication of a 3-dimensional superalgebra. We consider first the algebra $A_{1}$.

Proposition 3.2. Every non-trivial 3-dimensional superalgebra, where the even part is the 2-dimensional algebra $A_{1}$ is isomorphic to one of the following pairwise nonisomorphic 3-dimensional superalgebras $A_{1 \mid 1}=\mathbb{K}[x, y] /\left(x^{2}, y^{2}, x y\right)$ and $A_{1 \mid 2}=\mathbb{K}[x, y] /\left(x^{2}, y^{2}-x, x y\right)$, where $\operatorname{deg}(x)=0$ and $\operatorname{deg}(y)=1$.

Proof. We set

$$
x \cdot y=\alpha y, \quad y \cdot x=\beta y, \quad y \cdot y=\gamma e_{1}^{0}+\sigma x, \quad \text { with } \quad \alpha, \beta, \gamma, \sigma \in \mathbb{K} .
$$

We have $x \cdot(y \cdot y)=\gamma x,(x \cdot y) \cdot y=\alpha \gamma e_{1}^{0}+\alpha \sigma x,(y \cdot y) \cdot x=\gamma x$ and $y \cdot(y \cdot x)=\beta \gamma e_{1}^{0}+\beta \sigma x$.

According to associativity and by identification, we obtain $\alpha \gamma=0, \alpha \sigma=\gamma, \beta \gamma=0, \beta \sigma=\gamma$. Then $\gamma=0$ and $y \cdot y=\sigma x$.

Moreover, $x \cdot(x \cdot y)=(x \cdot x) \cdot y=0$ and $(y \cdot x) \cdot x=y \cdot(x \cdot x)=0$ lead to $\alpha=\beta=0$. Finally, it remains to fix $\sigma$.

If $\sigma=0$, we obtain the superalgebra defined by $A_{1 \mid 1}$. If $\sigma \neq 0$, then we have the superalgebra which is given by the multiplication $\mu_{\sigma}$ defined as $\mu_{\sigma}(x \otimes x)=0, \mu_{\sigma}(y \otimes x)=0, \mu_{\sigma}(x \otimes y)=0$, $\mu_{\sigma}(y \otimes y)=\sigma x, \sigma \neq 0$. It is isomorphic to $A_{1 \mid 2}(\sigma=1)$.

For the second algebra $A_{2}$, we obtain three possible extensions as a 3-dimensional superalgebra.

Proposition 3.3. Every non-trivial 3-dimensional superalgebra where the even part is the 2-dimensional algebra $A_{2}$, is isomorphic to one of the following pairwise nonisomorphic 3dimensional superalgebras $A_{2 \mid 1}=\mathbb{K}[x, y] /\left(x^{2}-x, y^{2}-x, x y-y\right), A_{2 \mid 2}=\mathbb{K}\langle x, y\rangle /\left(x^{2}-x, y^{2}\right.$, $x y-y, y x)$ and $A_{2 \mid 3}=\mathbb{K}[x, y] /\left(x^{2}-x, y^{2}, x y-y\right)$, where $\operatorname{deg}(x)=0$ and $\operatorname{deg}(y)=1$.

Proof. By associativity conditions $(x \cdot x) \cdot y=x \cdot(x \cdot y)$ and $y \cdot(x \cdot x)=(y \cdot x) \cdot x$, it follows that $\alpha$ and $\beta$ may have only values 0 or 1 .

Similarly, conditions $x \cdot(y \cdot y)=(x \cdot y) \cdot y$ and $y \cdot(y \cdot x)=(y \cdot y) \cdot x$ lead to

$$
\alpha \gamma=0, \quad \alpha \sigma=\gamma+\sigma, \quad \beta \gamma=0, \quad \beta \sigma=\gamma+\sigma .
$$

We discuss the two cases $\gamma=0$ and $\gamma \neq 0$.

1. If $\gamma=0$, then the system (3.1) reduces to $\sigma(\alpha-1)=0$ and $\sigma(\beta-1)=0$. So, we deduce two subcases:

a) If $\sigma \neq 0$, then $\alpha=\beta=1$. We obtain superalgebras which are isomorphic to $A_{2 \mid 1}(\sigma=1)$.

b) If $\sigma=0$, we study two cases $\alpha=\beta$ and $\alpha \neq \beta$, 
i) If $\alpha=\beta$, that means $(\alpha, \beta) \in\{(0,0),(1,1)\}$. Then, we obtain two superalgebras isomorphic to $A_{2 \mid 3}$.

ii) If $\alpha \neq \beta$, that means $(\alpha, \beta) \in\{(0,1),(1,0)\}$. Then, we obtain superalgebras which are isomorphic to $A_{2 \mid 2}$.

2. If $\gamma \neq 0$. Then, the system (3.1) reduces to $\alpha=\beta=0$ and $\sigma=-\gamma$. So, we have superalgebras with multiplications $\mu_{\gamma}$ defined as

$$
\mu_{\gamma}(x \otimes x)=x, \quad \mu_{\gamma}(y \otimes x)=0, \quad \mu_{\gamma}(x \otimes y)=0, \quad \mu_{\gamma}(y \otimes y)=\gamma(1-x),
$$

which are isomorphic to the superalgebra $A_{2 \mid 1}$.

\subsection{Superbialgebras and Hopf superalgebras}

Now, we construct superbialgebra structures associated to the five superalgebras found above.

Proposition 3.4. There is no 3-dimensional superbialgebra, with $\operatorname{dim} A_{0}=2$, associated to superalgebras $A_{1 \mid 1}$ and $A_{1 \mid 2}$.

Proof. Assume $\Delta(x)=\alpha 1 \otimes 1+\beta 1 \otimes x+\gamma x \otimes 1+\sigma x \otimes x+\delta y \otimes y$ where $\alpha, \beta, \gamma, \sigma, \delta \in \mathbb{K}$. Since we have $x^{2}=0$, the compatibility condition $\varepsilon(\mu(x \otimes x))=\varepsilon(x) \varepsilon(x)$, implies $\varepsilon(x)=0$.

In one hand, the condition $(\varepsilon \otimes \mathrm{id})(\Delta(x))=(\mathrm{id} \otimes \varepsilon)(\Delta(x))=x$ implies that $\alpha=0$ and $\beta=\gamma=1$. In the other hand, the compatibility condition $\Delta \circ \mu(x \otimes x)=(\mu \otimes \mu) \circ(\mathrm{id} \otimes \tau \otimes$ id $) \circ(\Delta \otimes \Delta)(x \otimes x)=0$, leads to $\beta \gamma=0$. Therefore we have a contradiction.

In the sequel, we consider the superalgebra structures defined by $\mu_{2 \mid 1}, \mu_{2 \mid 2}$ and $\mu_{2 \mid 3}$. We denote by $A_{i \mid j}^{k}$ the superbialgebra $\left(A, \mu_{i \mid j}, \eta, \Delta_{i \mid j}^{k}, \varepsilon_{i \mid j}^{k}\right)$. We have $A^{\text {cop }}=\left(A, \mu, \eta, \Delta^{\prime}, \varepsilon\right)$ where $\Delta^{\prime}=\Delta \circ \tau$ and $\tau$ is the superflip. By direct calculation, we obtain the following pairwise non-isomorphic superbialgebras. For all of them we have $\Delta_{i \mid j}^{k}(1)=1 \otimes 1$.

For superalgebra $A_{2 \mid 1}$, we have

1) $A_{2 \mid 1}^{1}$ with $\Delta_{2 \mid 1}^{1}(x)=1 \otimes x+x \otimes 1-x \otimes x, \Delta_{2 \mid 1}^{1}(y)=y \otimes 1+1 \otimes y-y \otimes x, \varepsilon_{2 \mid 1}^{1}(x)=0$;

2) $\left(A_{2 \mid 1}^{1}\right)^{\text {cop }}$.

For superalgebra $A_{2 \mid 2}$, we have

1) $A_{2 \mid 2}^{1}$ with $\Delta_{2 \mid 2}^{1}(x)=1 \otimes x+x \otimes 1-x \otimes x, \Delta_{2 \mid 2}^{1}(y)=1 \otimes y+y \otimes 1-y \otimes x-x \otimes y, \varepsilon_{2 \mid 2}^{1}(x)=0$;

2) $A_{2 \mid 2}^{2}$ with $\Delta_{2 \mid 2}^{2}(x)=1 \otimes x+x \otimes 1-x \otimes x+y \otimes y, \Delta_{2 \mid 2}^{2}(y)=1 \otimes y+y \otimes 1-y \otimes x-x \otimes y$, $\varepsilon_{2 \mid 2}^{2}(x)=0$;

3) $A_{2 \mid 2}^{3}$ with $\Delta_{2 \mid 2}^{3}(x)=x \otimes x, \Delta_{2 \mid 2}^{3}(y)=y \otimes x+x \otimes y, \varepsilon_{2 \mid 2}^{3}(x)=1$;

4) $A_{2 \mid 2}^{4}$ with $\Delta_{2 \mid 2}^{4}(x)=x \otimes x+y \otimes y, \Delta_{2 \mid 2}^{4}(y)=y \otimes x+x \otimes y, \varepsilon_{2 \mid 2}^{4}(x)=1$.

For superalgebra $A_{2 \mid 3}$, we have

1) $A_{2 \mid 3}^{1}$ with $\Delta_{2 \mid 3}^{1}(x)=1 \otimes x+x \otimes 1-x \otimes x, \Delta_{2 \mid 3}^{1}(y)=1 \otimes y+y \otimes 1-x \otimes y-y \otimes x, \varepsilon_{2 \mid 3}^{1}(x)=0$;

2) $A_{2 \mid 3}^{2}$ with $\Delta_{2 \mid 3}^{2}(x)=1 \otimes x+x \otimes 1-x \otimes x, \Delta_{2 \mid 3}^{2}(y)=1 \otimes y+y \otimes 1-x \otimes y, \varepsilon_{2 \mid 3}^{2}(x)=0$;

3) $\left(A_{2 \mid 3}^{2}\right)^{\text {cop }}$

4) $A_{2 \mid 3}^{4}$ with $\Delta_{2 \mid 3}^{4}(x)=1 \otimes x+x \otimes 1-x \otimes x, \Delta_{2 \mid 3}^{4}(y)=y \otimes 1+1 \otimes y, \varepsilon_{2 \mid 3}^{4}(x)=0$;

5) $A_{2 \mid 3}^{5}$ with $\Delta_{2 \mid 3}^{5}(x)=x \otimes x, \Delta_{2 \mid 3}^{5}(y)=y \otimes x+x \otimes y, \varepsilon_{2 \mid 3}^{5}(x)=1$. 
Theorem 3.5. Every non-trivial 3-dimensional superbialgebra with $\operatorname{dim} A_{0}=2$ is isomorphic to one of the following 3-dimensional pairwise non-isomorphic superbialgebras, which are defined above,

$$
A_{2 \mid 1}^{1}, \quad\left(A_{2 \mid 1}^{1}\right)^{\mathrm{cop}}, \quad A_{2 \mid 2}^{k} \quad(k=1, \ldots, 4), \quad A_{2 \mid 3}^{1}, \quad A_{2 \mid 3}^{2}, \quad\left(A_{2 \mid 3}^{2}\right)^{\mathrm{cop}}, \quad A_{2 \mid 3}^{4}, \quad A_{2 \mid 3}^{5} .
$$

Theorem 3.6. There is no non-trivial 3-dimensional Hopf superalgebra.

Proof. We check that no one of the superbialgebras may carry a structure of Hopf superalgebra. Let $S$ be an antipode of one of the 3-dimensional superbialgebras defined above. Assume that

$$
S(1)=1, \quad S(x)=\lambda_{1} 1+\lambda_{2} x, \quad S(y)=\lambda_{3} y,
$$

and $S$ satisfies the identity $\mu \circ(S \otimes$ id $) \circ \Delta=\mu \circ(\mathrm{id} \otimes S) \circ \Delta=\eta \circ \varepsilon$.

We apply the identity to $x$ and study two cases.

Case 1: $\varepsilon(x)=0$. For all 3-dimensional superalgebras in this case, we have

$$
\Delta(x)=1 \otimes x+x \otimes 1-x \otimes x+\alpha y \otimes y, \quad \text { such that } \quad \alpha=0 \quad \text { or } \quad 1 .
$$

In one hand, we have,

$$
\begin{aligned}
& \mu \circ\left(S \otimes \mathrm{id}_{A}\right) \circ \Delta(x)=\mu \circ(1 \otimes x+x \otimes 1-S(x) \otimes x+\alpha S(y) \otimes y) \\
& \quad=x+\left(\lambda_{1} x+\lambda_{2} x\right)-\lambda_{1} x-\lambda_{2} x=\lambda_{1} 1+\left(1-\lambda_{1}\right) x .
\end{aligned}
$$

In the other hand, we have $\eta \circ \varepsilon(x)=0$. Therefore, we have a contradiction.

Case 2: $\varepsilon(x)=1$. In this case, $\Delta(x)$ must have the form

$$
\Delta(x)=x \otimes x+\alpha y \otimes y, \quad \text { such that } \quad \alpha=0 \quad \text { or } \quad 1 .
$$

Then, on one hand, we have,

$$
\mu \circ\left(S \otimes \operatorname{id}_{A}\right) \circ \Delta(x)=\mu \circ(S(x) \otimes x+\alpha S(y) \otimes y)=\left(\lambda_{1}+\lambda_{2}\right) x .
$$

On the other hand, we have $\eta \circ \varepsilon(x)=1$. So we have a contradiction.

\section{Classification of 4-dimensional superbialgebras and Hopf superalgebras}

Let $A=A_{0} \oplus A_{1}$ be a 4-dimensional superbialgebra. Then one has to consider four cases for $n_{0}=\operatorname{dim} A_{0}$. If $n_{0}=1$, the superbialgebras are connected, then it is covered by Proposition 2.3. If $n_{0}=4$, then they correspond to trivial superbialgebras. In the sequel, we discuss cases $n_{0}=2$ and $n_{0}=3$.

\subsection{4-dimensional algebras}

In the following, we recall the classification of 4-dimensional algebras given by Gabriel in [17] and the classification of 4-dimensional superalgebras with $n_{0}=2$ and $n_{0}=3$ provided by Armour, Chen and Zhang in [7].

Theorem 4.1. The following algebras are pairwise non-isomorphic and every 4-dimensional algebras is isomorphic to one of these 19 algebras:

1) $\mathbb{K} \times \mathbb{K} \times \mathbb{K} \times \mathbb{K}$,

2) $\mathbb{K} \times \mathbb{K} \times \mathbb{K}[x] / x^{2}$, 
3) $\mathbb{K}[x] / x^{2} \times \mathbb{K}[y] / y^{2}$,

4) $\mathbb{K} \times \mathbb{K}[x] / x^{3}$,

5) $\mathbb{K}[x] / x^{4}$,

6) $\mathbb{K} \times \mathbb{K}[x, y] /(x, y)^{2}$,

7) $\mathbb{K}[x, y] /\left(x^{2}, y^{2}\right)$,

8) $\mathbb{K}[x, y] /\left(x^{3}, x y, y^{2}\right)$,

9) $\mathbb{K}[x, y, z] /(x, y, z)^{2}$,

10) $M_{2}=\left(\begin{array}{ll}\mathbb{K} & \mathbb{K} \\ \mathbb{K} & \mathbb{K}\end{array}\right)$,

11) $\left\{\left(\begin{array}{llll}a & 0 & 0 & 0 \\ 0 & a & 0 & d \\ c & 0 & b & 0 \\ 0 & 0 & 0 & b\end{array}\right) / a, b, c, d \in \mathbb{K}\right\}$,

12) $\Lambda \mathbb{K}^{2}=$ exterior algebra of $\mathbb{K}^{2}$,

13) $\mathbb{K} \times\left(\begin{array}{cc}\mathbb{K} & \mathbb{K} \\ 0 & \mathbb{K}\end{array}\right)$,

14) $\left\{\left(\begin{array}{lll}a & 0 & 0 \\ c & a & 0 \\ d & 0 & b\end{array}\right) / a, b, c, d \in \mathbb{K}\right\}$,

15) $\left\{\left(\begin{array}{lll}a & c & d \\ 0 & a & 0 \\ 0 & 0 & b\end{array}\right) / a, b, c, d \in \mathbb{K}\right\}$,

16) $\mathbb{K}[x, y] /\left(x^{2}, y^{2}, y x\right)$,

17) $\left\{\left(\begin{array}{lll}a & 0 & 0 \\ 0 & a & 0 \\ c & d & b\end{array}\right) / a, b, c, d \in \mathbb{K}\right\}$,

18) $\mathbb{K}\langle x, y\rangle /\left(x^{2}, y^{2}, y x-\lambda x y\right)$, for $\lambda \neq-1,0,1$,

19) $\mathbb{K}\langle x, y\rangle /\left(y^{2}, x^{2}+y x, x y+y x\right)$,

where $\mathbb{K}\langle x, y\rangle$ is the free associative algebra generated by $x$ and $y$.

\subsection{Superalgebras with $\operatorname{dim}\left(A_{0}\right)=3$}

Let $\left\{e_{1}^{0}, e_{2}^{0}, e_{3}^{0}, e_{1}^{1}\right\}$ be a basis of the superalgebra $A, A=A_{0} \oplus A_{1}$, such that $A_{0}=\operatorname{span}\left\{e_{1}^{0}, e_{2}^{0}, e_{3}^{0}\right\}$, $A_{1}=\operatorname{span}\left\{e_{1}^{1}\right\}$ and $e_{1}^{0}$ be the unit element of the superalgebra. We recall the multiplication of all possible graduation obtained from a fixed algebra, we denote by $\mu_{i \mid j}$ the multiplication of each 4-dimensional superalgebra in the case $\operatorname{dim} A_{0}=3$. We preserve the notation used in [7], a superalgebra $i \mid j$ means the $j^{\text {th }}$ superalgebra obtained by a fixed $i^{\text {th }} 4$-dimensional algebra.

Proposition 4.2 ([7]). Let $\mathbb{K}$ be an algebraically closed field of characteristic 0 , suppose that $A$ is a 4-dimensional superalgebra with $\operatorname{dim} A_{0}=3$. Then $A$ is isomorphic to one of the superalgebra in the following pairwise nonisomorphic families:

$\mathbf{1} \mid \mathbf{1}: \mathbb{K} \times \mathbb{K} \times \mathbb{K} \times \mathbb{K}, e_{1}^{0}=(1,1,1,1), e_{2}^{0}=(1,0,0,0), e_{3}^{0}=(0,0,1,1), e_{1}^{1}=(0,0,1,-1) ;$

$\mathbf{2} \mid \mathbf{1}: \mathbb{K} \times \mathbb{K} \times \mathbb{K}[x] / x^{2}, e_{1}^{0}=(1,1,1), e_{2}^{0}=(1,0,0), e_{3}^{0}=(0,1,0), e_{1}^{1}=(0,0, x) ;$

$\mathbf{2} \mid \mathbf{2}: \mathbb{K} \times \mathbb{K} \times \mathbb{K}[x] / x^{2}, e_{1}^{0}=(1,1,1), e_{2}^{0}=(1,1,0), e_{3}^{0}=(0,0, x), e_{1}^{1}=(1,-1,0)$;

3|1: $\mathbb{K}[x] / x^{2} \times \mathbb{K}[y] / y^{2}, e_{1}^{0}=(1,1), e_{2}^{0}=(1,0), e_{3}^{0}=(x, 0), e_{1}^{1}=(0, y) ;$

4|1: $\mathbb{K} \times \mathbb{K}[x] / x^{3}, e_{1}^{0}=(1,1), e_{2}^{0}=(1,0), e_{3}^{0}=\left(0, x^{2}\right), e_{1}^{1}=(0, x)$;

6|1: $\mathbb{K} \times \mathbb{K}[x, y] /(x, y)^{2}: e_{1}^{0}=(1,1), e_{2}^{0}=(1,0), e_{3}^{0}=(0, x), e_{1}^{1}=(0, y)$;

$\mathbf{7} \mid \mathbf{1}: \mathbb{K}[x, y] /\left(x^{2}, y^{2}\right), e_{1}^{0}=1, e_{2}^{0}=x+y, e_{3}^{0}=x y, e_{1}^{1}=x-y ;$ 
8|1: $\mathbb{K}[x, y] /\left(x^{3}, x y, y^{2}\right), e_{1}^{0}=1, e_{2}^{0}=x, e_{3}^{0}=x^{2}, e_{1}^{1}=y ;$

8|2: $\mathbb{K}[x, y] /\left(x^{3}, x y, y^{2}\right), e_{1}^{0}=1, e_{2}^{0}=x^{2}, e_{3}^{0}=y, e_{1}^{1}=x ;$

$\mathbf{9} \mid \mathbf{1}: \mathbb{K}[x, y, z] /(x, y, z)^{2}, e_{1}^{0}=1, e_{2}^{0}=x, e_{3}^{0}=y, e_{1}^{1}=z ;$

$\begin{aligned} \mathbf{1 1} \mid \mathbf{1}: & \left\{\left(\begin{array}{llll}a & 0 & 0 & 0 \\ 0 & a & 0 & d \\ c & 0 & b & 0 \\ 0 & 0 & 0 & b\end{array}\right) / a, b, c, d \in \mathbb{K}\right\}, e_{1}^{0}=\left(\begin{array}{llll}1 & 0 & 0 & 0 \\ 0 & 1 & 0 & 0 \\ 0 & 0 & 1 & 0 \\ 0 & 0 & 0 & 1\end{array}\right), e_{2}^{0}=\left(\begin{array}{llll}1 & 0 & 0 & 0 \\ 0 & 1 & 0 & 0 \\ 0 & 0 & 0 & 0 \\ 0 & 0 & 0 & 0\end{array}\right), \\ e_{3}^{0} & =\left(\begin{array}{llll}0 & 0 & 0 & 0 \\ 0 & 0 & 0 & 1 \\ 0 & 0 & 0 & 0 \\ 0 & 0 & 0 & 0\end{array}\right), e_{1}^{1}=\left(\begin{array}{llll}0 & 0 & 0 & 0 \\ 0 & 0 & 0 & 0 \\ 1 & 0 & 0 & 0 \\ 0 & 0 & 0 & 0\end{array}\right) ;\end{aligned}$

13|1: $\mathbb{K} \times\left(\begin{array}{cc}\mathbb{K} & \mathbb{K} \\ 0 & \mathbb{K}\end{array}\right)=\left\{\left(a,\left(\begin{array}{ll}b & c \\ 0 & d\end{array}\right)\right) / a, b, c, d \in \mathbb{K}\right\}, e_{1}^{0}=\left(1,\left(\begin{array}{ll}1 & 0 \\ 0 & 1\end{array}\right)\right)$,

$e_{2}^{0}=\left(0,\left(\begin{array}{ll}1 & 0 \\ 0 & 0\end{array}\right)\right), e_{3}^{0}=\left(0,\left(\begin{array}{ll}0 & 0 \\ 0 & 1\end{array}\right)\right), e_{1}^{1}=\left(0,\left(\begin{array}{ll}0 & 1 \\ 0 & 0\end{array}\right)\right)$;

$\mathbf{1 4} \mid \mathbf{1}:\left\{\left(\begin{array}{lll}a & 0 & 0 \\ c & a & 0 \\ d & 0 & b\end{array}\right) / a, b, c, d \in \mathbb{K}\right\}, e_{1}^{0}=\left(\begin{array}{lll}1 & 0 & 0 \\ 0 & 1 & 0 \\ 0 & 0 & 1\end{array}\right), e_{2}^{0}=\left(\begin{array}{lll}1 & 0 & 0 \\ 0 & 1 & 0 \\ 0 & 0 & 0\end{array}\right)$,

$e_{3}^{0}=\left(\begin{array}{lll}0 & 0 & 0 \\ 0 & 0 & 0 \\ 1 & 0 & 0\end{array}\right), e_{1}^{1}=\left(\begin{array}{lll}0 & 0 & 0 \\ 1 & 0 & 0 \\ 0 & 0 & 0\end{array}\right)$

14|2: $\left\{\left(\begin{array}{ccc}a & 0 & 0 \\ c & a & 0 \\ d & 0 & b\end{array}\right) / a, b, c, d \in \mathbb{K}\right\}, e_{1}^{0}=\left(\begin{array}{lll}1 & 0 & 0 \\ 0 & 1 & 0 \\ 0 & 0 & 1\end{array}\right), e_{2}^{0}=\left(\begin{array}{lll}1 & 0 & 0 \\ 0 & 1 & 0 \\ 0 & 0 & 0\end{array}\right)$,

$e_{3}^{0}=\left(\begin{array}{lll}0 & 0 & 0 \\ 1 & 0 & 0 \\ 0 & 0 & 0\end{array}\right), e_{1}^{1}=\left(\begin{array}{ccc}0 & 0 & 0 \\ 0 & 0 & 0 \\ 1 & 0 & 0\end{array}\right)$;

15|1: $\left\{\left(\begin{array}{lll}a & c & d \\ 0 & a & 0 \\ 0 & 0 & b\end{array}\right) / a, b, c, d \in \mathbb{K}\right\}, e_{1}^{0}=\left(\begin{array}{lll}1 & 0 & 0 \\ 0 & 1 & 0 \\ 0 & 0 & 1\end{array}\right), e_{2}^{0}=\left(\begin{array}{lll}1 & 0 & 0 \\ 0 & 1 & 0 \\ 0 & 0 & 0\end{array}\right)$,

$e_{3}^{0}=\left(\begin{array}{lll}0 & 0 & 1 \\ 0 & 0 & 0 \\ 0 & 0 & 0\end{array}\right), e_{1}^{1}=\left(\begin{array}{ccc}0 & 1 & 0 \\ 0 & 0 & 0 \\ 0 & 0 & 0\end{array}\right)$

15|2: $\left\{\left(\begin{array}{lll}a & c & d \\ 0 & a & 0 \\ 0 & 0 & b\end{array}\right) / a, b, c, d \in \mathbb{K}\right\}, e_{1}^{0}=\left(\begin{array}{lll}1 & 0 & 0 \\ 0 & 1 & 0 \\ 0 & 0 & 1\end{array}\right), e_{2}^{0}=\left(\begin{array}{lll}1 & 0 & 0 \\ 0 & 1 & 0 \\ 0 & 0 & 0\end{array}\right)$,

$e_{3}^{0}=\left(\begin{array}{lll}0 & 1 & 0 \\ 0 & 0 & 0 \\ 0 & 0 & 0\end{array}\right), e_{1}^{1}=\left(\begin{array}{ccc}0 & 0 & 1 \\ 0 & 0 & 0 \\ 0 & 0 & 0\end{array}\right)$

$\mathbf{1 7} \mid \mathbf{1}:\left\{\left(\begin{array}{lll}a & 0 & 0 \\ 0 & a & 0 \\ c & d & b\end{array}\right) / a, b, c, d \in \mathbb{K}\right\}, e_{1}^{0}=\left(\begin{array}{lll}1 & 0 & 0 \\ 0 & 1 & 0 \\ 0 & 0 & 1\end{array}\right), e_{2}^{0}=\left(\begin{array}{lll}1 & 0 & 0 \\ 0 & 1 & 0 \\ 0 & 0 & 0\end{array}\right)$,

$e_{3}^{0}=\left(\begin{array}{lll}0 & 0 & 0 \\ 0 & 0 & 0 \\ 1 & 0 & 0\end{array}\right), e_{1}^{1}=\left(\begin{array}{lll}0 & 0 & 0 \\ 0 & 0 & 0 \\ 0 & 1 & 0\end{array}\right)$. 


\subsection{Superbialgebras and Hopf superalgebras with $\operatorname{dim}\left(A_{0}\right)=3$}

We look for all possible superbialgebras which could be obtained from a fixed superalgebra. The computation are done using a computer algebra system. For the convenience of the presentation, we only summarize the results in the following proposition. The notations and details are collected in Appendix A.1.

Proposition 4.3. Let $(A, \mu, \eta, \Delta, \varepsilon)$ be a 4-dimensional superbialgebra with $\operatorname{dim} A_{0}=3$. Then $A$ is isomorphic to one of the superbialgebra in the following pairwise nonisomorphic families:

\begin{tabular}{cl}
\hline superalgebra & associated superbialgebras \\
\hline $\mathbf{1} \mid \mathbf{1}$ & $\left(A, \mu_{1 \mid 1}, \eta, \Delta_{1 \mid 1}^{k}, \varepsilon_{1 \mid 1}^{k}\right), k=1, \ldots, 12$ \\
$\mathbf{2} \mid \mathbf{1}$ & $\left(A, \mu_{2 \mid 1}, \eta, \Delta_{2 \mid 1}^{k}, \varepsilon_{2 \mid 1}^{k}\right), k=1, \ldots, 22$ \\
$\mathbf{4} \mid \mathbf{1}$ & $\left(A, \mu_{4 \mid 1}, \eta, \Delta_{4 \mid 1}^{k}, \varepsilon_{4 \mid 1}^{k}\right), k=1,2,3$ \\
$\mathbf{6} \mid \mathbf{1}$ & $\left(A, \mu_{6 \mid 1}, \eta, \Delta_{6 \mid 1}^{k}, \varepsilon_{6 \mid 1}^{k}\right), k=1, \ldots, 18$ \\
$\mathbf{1 3} \mid \mathbf{1}$ & $\left(A, \mu_{13 \mid 1}, \eta, \Delta_{13 \mid 1}^{k}, \varepsilon_{13 \mid 1}^{k}\right), k=1, \ldots, 21$ \\
$\mathbf{1 4} \mid \mathbf{1}$ & $\left(A, \mu_{14 \mid 1}, \eta, \Delta_{14 \mid 1}^{k}, \varepsilon_{14 \mid 1}^{k}\right), k=1, \ldots, 9$ \\
$\mathbf{1 4} \mid \mathbf{2}$ & $\left(A, \mu_{14 \mid 2}, \eta, \Delta_{14 \mid 2}^{k}, \varepsilon_{14 \mid 2}^{k}\right), k=1, \ldots, 4$ \\
$\mathbf{1 5} \mid \mathbf{1}$ & $\left(A, \mu_{15 \mid 1}, \eta, \Delta_{15 \mid 1}^{k}, \varepsilon_{15 \mid 1}^{k}\right), k=1, \ldots, 9$ \\
$\mathbf{1 5} \mid \mathbf{2}$ & $\left(A, \mu_{15 \mid 2}, \eta, \Delta_{15 \mid 2}^{k}, \varepsilon_{15 \mid 2}^{k}\right), k=1, \ldots, 4$ \\
$\mathbf{1 7} \mid \mathbf{1}$ & $\left(A, \mu_{17 \mid 1}, \eta, \Delta_{17 \mid 1}^{k}, \varepsilon_{17 \mid 1}^{k}\right), k=1, \ldots, 11$ \\
\hline
\end{tabular}

Remark 4.4. There is no 4-dimensional superbialgebra with $\operatorname{dim} A_{0}=3$ and underlying multiplications $\mu_{2 \mid 2}, \mu_{3 \mid 1}, \mu_{7 \mid 1}, \mu_{8 \mid 1}, \mu_{8 \mid 2}, \mu_{9 \mid 1}, \mu_{11 \mid 1}$.

Now, we look for Hopf superalgebra structures. For a fixed superbialgebra defined above, we add the antipode's property. It turns out that there exists only one non-trivial 4-dimensional Hopf superalgebra in the case $\operatorname{dim} A_{0}=3$. It corresponds to the algebra (1|1) and comultiplication $\Delta_{1 \mid 1}^{2}$, where $A=\mathbb{K} \times \mathbb{K} \times \mathbb{K} \times \mathbb{K}$. We set $x=e_{1}^{0}-2 e_{2}^{0}-e_{3}^{0}=(-1,1,0,0)$ and $y=e_{1}^{1}=(0,0,1,-1)$ with $\operatorname{deg}(x)=0, \operatorname{deg}(y)=1$. It leads to basis $\left\{1, x, x^{2}, y\right\}$ and the algebra may be written as $\mathbb{K}[x, y] /\left(x^{2}+y^{2}-1, x y\right)$ with $\operatorname{deg}(x)=0$ and $\operatorname{deg}(y)=1$.

Proposition 4.5. Every non-trivial 4-dimensional Hopf superalgebra where $\operatorname{dim} A_{0}=3$ is isomorphic to the 4-dimensional Hopf superalgebra $\mathbb{K}[x, y] /\left(x^{2}+y^{2}-1, x y\right)$ with $\operatorname{deg}(x)=0$, $\operatorname{deg}(y)=1$ and such that

$$
\begin{array}{lrl}
\Delta(x)=x \otimes x-\alpha y \otimes y, & \varepsilon(x)=1, & S(x)=x, \\
\Delta(y)=x \otimes y+y \otimes x, & \varepsilon(y)=0, & S(y)=\alpha y .
\end{array}
$$

where $\alpha$ is a primitive $4^{\text {th }}$ root of unity.

\subsection{Superalgebras with $\operatorname{dim}\left(A_{0}\right)=2$}

Let $\left\{e_{1}^{0}, e_{2}^{0}, e_{1}^{1}, e_{2}^{1}\right\}$ be a basis of the underlying superspace $A$, such that $\left\{e_{1}^{0}, e_{2}^{0}\right\}$ is a basis of the even part, $\left\{e_{1}^{1}, e_{2}^{1}\right\}$ a basis of the odd part and $e_{1}^{0}$ is the unit of the superalgebra.

Proposition 4.6 ([7]). Let $\mathbb{K}$ be an algebraically closed field, suppose that $A$ is a 4-dimensional superalgebra with $\operatorname{dim} A_{0}=2$. Then $A$ is isomorphic to one of the superalgebra in the following pairwise non-isomorphic families:

$\mathbf{1} \mid \mathbf{2}: \mathbb{K} \times \mathbb{K} \times \mathbb{K} \times \mathbb{K}, e_{1}^{0}=(1,1,1,1), e_{2}^{0}=(1,1,0,0), e_{1}^{1}=(1,-1,0,0), e_{2}^{1}=(0,0,1,-1) ;$ 
$\mathbf{2} \mid \mathbf{3}: \mathbb{K} \times \mathbb{K} \times \mathbb{K}[x] / x^{2}, e_{1}^{0}=(1,1,1), e_{2}^{0}=(1,1,0), e_{1}^{1}=(1,-1,0), e_{2}^{1}=(0,0, x) ;$

3|2: $\mathbb{K}[x] / x^{2} \times \mathbb{K}[y] / y^{2}, e_{1}^{0}=(1,1), e_{2}^{0}=(1,0), e_{1}^{1}=(x, 0), e_{2}^{1}=(0, y)$;

$\mathbf{3} \mid \mathbf{3}: \mathbb{K}[x] / x^{2} \times \mathbb{K}[y] / y^{2}, e_{1}^{0}=(1,1), e_{2}^{0}=(x, y), e_{1}^{1}=(1,-1), e_{2}^{1}=(x,-y) ;$

$\mathbf{5} \mid \mathbf{1}: \mathbb{K}[x] / x^{4}, e_{1}^{0}=1, e_{2}^{0}=x^{2}, e_{1}^{1}=x, e_{2}^{1}=x^{3} ;$

6|2: $\mathbb{K} \times \mathbb{K}[x, y] /(x, y)^{2}, e_{1}^{0}=(1,1), e_{2}^{0}=(1,0), e_{1}^{1}=(0, x), e_{2}^{1}=(0, y) ;$

$\mathbf{7} \mid \mathbf{2}: \mathbb{K}[x, y] /\left(x^{2}, y^{2}\right), e_{1}^{0}=1, e_{2}^{0}=x, e_{1}^{1}=y, e_{2}^{1}=x y ;$

7|3: $\mathbb{K}[x, y] /\left(x^{2}, y^{2}\right), e_{1}^{0}=1, e_{2}^{0}=x y, e_{1}^{1}=x, e_{2}^{1}=y ;$

8|3: $\mathbb{K}[x, y] /\left(x^{3}, x y, y^{2}\right), e_{1}^{0}=1, e_{2}^{0}=x^{2}, e_{1}^{1}=x, e_{2}^{1}=y$;

$\mathbf{9} \mid \mathbf{2}: \mathbb{K}[x, y, z] /(x, y, z)^{2}, e_{1}^{0}=1, e_{2}^{0}=x, e_{1}^{1}=y, e_{2}^{1}=z ;$

10|1: $M_{2}=\left(\begin{array}{ll}\mathbb{K} & \mathbb{K} \\ \mathbb{K} & \mathbb{K}\end{array}\right)=\left\{\left(\begin{array}{ll}a & b \\ c & d\end{array}\right) / a, b, c, d \in \mathbb{K}\right\}, e_{1}^{0}=\left(\begin{array}{ll}1 & 0 \\ 0 & 1\end{array}\right), e_{2}^{0}=\left(\begin{array}{ll}1 & 0 \\ 0 & 0\end{array}\right)$,

$e_{1}^{1}=\left(\begin{array}{ll}0 & 1 \\ 0 & 0\end{array}\right), e_{2}^{1}=\left(\begin{array}{ll}0 & 0 \\ 1 & 0\end{array}\right)$;

$\mathbf{1 1} \mid \mathbf{2}:\left\{\left(\begin{array}{cccc}a & 0 & 0 & 0 \\ 0 & a & 0 & d \\ c & 0 & b & 0 \\ 0 & 0 & 0 & b\end{array}\right) / a, b, c, d \in \mathbb{K}\right\}, e_{1}^{0}=\left(\begin{array}{cccc}1 & 0 & 0 & 0 \\ 0 & 1 & 0 & 0 \\ 0 & 0 & 1 & 0 \\ 0 & 0 & 0 & 1\end{array}\right)$,

$e_{2}^{0}=\left(\begin{array}{cccc}1 & 0 & 0 & 0 \\ 0 & 1 & 0 & 0 \\ 0 & 0 & 0 & 0 \\ 0 & 0 & 0 & 0\end{array}\right), e_{1}^{1}=\left(\begin{array}{cccc}0 & 0 & 0 & 0 \\ 0 & 0 & 0 & 1 \\ 0 & 0 & 0 & 0 \\ 0 & 0 & 0 & 0\end{array}\right), e_{2}^{1}=\left(\begin{array}{cccc}0 & 0 & 0 & 0 \\ 0 & 0 & 0 & 0 \\ 1 & 0 & 0 & 0 \\ 0 & 0 & 0 & 0\end{array}\right)$

$\mathbf{1 1} \mid \mathbf{3}:\left\{\left(\begin{array}{cccc}a & 0 & 0 & 0 \\ 0 & a & 0 & d \\ c & 0 & b & 0 \\ 0 & 0 & 0 & b\end{array}\right) / a, b, c, d \in \mathbb{K}\right\}, e_{1}^{0}=\left(\begin{array}{cccc}1 & 0 & 0 & 0 \\ 0 & 1 & 0 & 0 \\ 0 & 0 & 1 & 0 \\ 0 & 0 & 0 & 1\end{array}\right)$,

$$
e_{2}^{0}=\left(\begin{array}{cccc}
0 & 0 & 0 & 0 \\
0 & 0 & 0 & 1 \\
1 & 0 & 0 & 0 \\
0 & 0 & 0 & 0
\end{array}\right), e_{1}^{1}=\left(\begin{array}{cccc}
1 & 0 & 0 & 0 \\
0 & 1 & 0 & 0 \\
0 & 0 & -1 & 0 \\
0 & 0 & 0 & -1
\end{array}\right), e_{2}^{1}=\left(\begin{array}{cccc}
0 & 0 & 0 & 0 \\
0 & 0 & 0 & -1 \\
1 & 0 & 0 & 0 \\
0 & 0 & 0 & 0
\end{array}\right) ;
$$

12|1: $\Lambda \mathbb{K}^{2}=\mathbb{K}\langle x, y\rangle /\left(x^{2}, y^{2}, x y+y x\right), e_{1}^{0}=1, e_{2}^{0}=x, e_{1}^{1}=y, e_{2}^{1}=x y$;

12|2: $\Lambda \mathbb{K}^{2}=\mathbb{K}\langle x, y\rangle /\left(x^{2}, y^{2}, x y+y x\right), e_{1}^{0}=1, e_{2}^{0}=x y, e_{1}^{1}=x, e_{2}^{1}=y$;

14|3: $\left\{\left(\begin{array}{lll}a & 0 & 0 \\ c & a & 0 \\ d & 0 & b\end{array}\right) / a, b, c, d \in \mathbb{K}\right\}, e_{1}^{0}=\left(\begin{array}{lll}1 & 0 & 0 \\ 0 & 1 & 0 \\ 0 & 0 & 1\end{array}\right), e_{2}^{0}=\left(\begin{array}{lll}1 & 0 & 0 \\ 0 & 1 & 0 \\ 0 & 0 & 0\end{array}\right)$, $e_{1}^{1}=\left(\begin{array}{lll}0 & 0 & 0 \\ 1 & 0 & 0 \\ 0 & 0 & 0\end{array}\right), e_{2}^{1}=\left(\begin{array}{ccc}0 & 0 & 0 \\ 0 & 0 & 0 \\ 1 & 0 & 0\end{array}\right)$

15 $\mid \mathbf{3 :}\left\{\left(\begin{array}{ccc}a & c & d \\ 0 & a & 0 \\ 0 & 0 & b\end{array}\right) / a, b, c, d \in \mathbb{K}\right\}, e_{1}^{0}=\left(\begin{array}{lll}1 & 0 & 0 \\ 0 & 1 & 0 \\ 0 & 0 & 1\end{array}\right), e_{2}^{0}=\left(\begin{array}{ccc}1 & 0 & 0 \\ 0 & 1 & 0 \\ 0 & 0 & 0\end{array}\right)$, $e_{1}^{1}=\left(\begin{array}{lll}0 & 1 & 0 \\ 0 & 0 & 0 \\ 0 & 0 & 0\end{array}\right), e_{2}^{1}=\left(\begin{array}{ccc}0 & 0 & 1 \\ 0 & 0 & 0 \\ 0 & 0 & 0\end{array}\right)$

16|1: $\mathbb{K}\langle x, y\rangle /\left(x^{2}, y^{2}, y x\right), e_{1}^{0}=1, e_{2}^{0}=x, e_{1}^{1}=y, e_{2}^{1}=x y$; 
16|2: $\mathbb{K}\langle x, y\rangle /\left(x^{2}, y^{2}, y x\right), e_{1}^{0}=1, e_{2}^{0}=y, e_{1}^{1}=x, e_{2}^{1}=x y$;

16|3: $\mathbb{K}\langle x, y\rangle /\left(x^{2}, y^{2}, y x\right), e_{1}^{0}=1, e_{2}^{0}=x y, e_{1}^{1}=x, e_{2}^{1}=y$;

$\mathbf{1 7} \mid \mathbf{2}:\left\{\left(\begin{array}{lll}a & 0 & 0 \\ 0 & a & 0 \\ c & d & b\end{array}\right) / a, b, c, d \in \mathbb{K}\right\}, e_{1}^{0}=\left(\begin{array}{lll}1 & 0 & 0 \\ 0 & 1 & 0 \\ 0 & 0 & 1\end{array}\right), e_{2}^{0}=\left(\begin{array}{lll}1 & 0 & 0 \\ 0 & 1 & 0 \\ 0 & 0 & 0\end{array}\right)$,

$$
e_{1}^{1}=\left(\begin{array}{ccc}
0 & 0 & 0 \\
0 & 0 & 0 \\
1 & 0 & 0
\end{array}\right), e_{2}^{1}=\left(\begin{array}{lll}
0 & 0 & 0 \\
0 & 0 & 0 \\
0 & 1 & 0
\end{array}\right)
$$

$(\mathbf{1 8} ; \lambda \mid \mathbf{1}): \mathbb{K}\langle x, y\rangle /\left(x^{2}, y^{2}, y x-\lambda x y\right)$, where $\lambda \in \mathbb{K}$ with $\lambda \neq-1,0,1$, $e_{1}^{0}=1, e_{2}^{0}=x, e_{1}^{1}=y, e_{2}^{1}=x y$;

$(\mathbf{1 8} ; \lambda \mid \mathbf{2}): \mathbb{K}\langle x, y\rangle /\left(x^{2}, y^{2}, y x-\lambda x y\right)$, where $\lambda \in \mathbb{K}$ with $\lambda \neq-1,0,1$, $e_{1}^{0}=1, e_{2}^{0}=x y, e_{1}^{1}=x, e_{2}^{1}=y$;

19|1: $\mathbb{K}\langle x, y\rangle /\left(y^{2}, x^{2}+y x, x y+y x\right), e_{1}^{0}=1, e_{2}^{0}=x y, e_{1}^{1}=x, e_{2}^{1}=y$.

\subsection{Superbialgebras and Hopf superalgebras with $\operatorname{dim}\left(A_{0}\right)=2$}

Now, we compute all superbialgebra structures associated to superalgebras described above where $\operatorname{dim} A_{0}=2$. We give fairly basic description of the results, all details are given in Appendix A.2. We denote by $\mu_{i \mid j}$ the multiplication in $A_{i \mid j}$ with respect to the basis described above.

Proposition 4.7. There is no 4-dimensional superbialgebra with $\operatorname{dim} A_{0}=2$ endowed with one of these multiplications $\mu_{1 \mid 2}, \mu_{3 \mid 3}, \mu_{5 \mid 1}, \mu_{7 \mid 2}, \mu_{7 \mid 3}, \mu_{8 \mid 3}, \mu_{10 \mid 1}, \mu_{1|| 3}, \mu_{12 \mid 1}, \mu_{16 \mid 1}, \mu_{16 \mid 2}, \mu_{16 \mid 3}$, $\mu_{18 \mid 1}, \mu_{18 \mid 2}, \mu_{19 \mid 1}$.

Proposition 4.8. Let $(A, \mu, \eta, \Delta, \varepsilon)$ be a 4-dimensional superbialgebra with $\operatorname{dim} A_{0}=2$, then $A$ is isomorphic to one of the superbialgebra in the following pairwise nonisomorphic families:

\begin{tabular}{cl}
\hline superalgebra & associated superbialgebras \\
\hline $\mathbf{2} \mid \mathbf{3}$ & $\left(A, \mu_{2 \mid 3}, \eta, \Delta_{2 \mid 3}^{k}, \varepsilon_{2 \mid 3}^{k}\right), k=1, \ldots, 4$ \\
$\mathbf{3} \mid \mathbf{2}$ & $\left(A, \mu_{3 \mid 2}, \eta, \Delta_{3 \mid 2}^{k}, \varepsilon_{3 \mid 2}^{k}\right), k=1, \ldots, 9$ \\
$\mathbf{6} \mid \mathbf{2}$ & $\left(A, \mu_{6 \mid 2}, \eta, \Delta_{6 \mid 2}^{k}, \varepsilon_{6 \mid 2}^{k}\right), k=1, \ldots, 11$ \\
$\mathbf{1 1} \mid \mathbf{2}$ & $\left(A, \mu_{11 \mid 2}, \eta, \Delta_{11 \mid 2}^{1}, \varepsilon_{11 \mid 2}^{1}\right)$ \\
$\mathbf{1 2} \mid \mathbf{2}$ & $\left(A, \mu_{12 \mid 2}, \eta, \Delta_{12 \mid 2}^{1}, \varepsilon_{12 \mid 2}^{1}\right)$ \\
$\mathbf{1 4} \mid \mathbf{3}$ & $\left(A, \mu_{14 \mid 3}, \eta, \Delta_{14 \mid 3}^{1}, \varepsilon_{14 \mid 3}^{1}\right), k=1, \ldots, 7$ \\
$\mathbf{1 5} \mid \mathbf{3}$ & $\left(A, \mu_{15 \mid 3}, \eta, \Delta_{15 \mid 3}^{1}, \varepsilon_{15 \mid 3}^{1}\right), k=1, \ldots, 7$ \\
$\mathbf{1 7} \mid \mathbf{2}$ & $\left(A, \mu_{17 \mid 2}, \eta, \Delta_{17 \mid 2}^{1}, \varepsilon_{17 \mid 2}^{1}\right), k=1,2$ \\
\hline
\end{tabular}

It turns out that the only superbialgebras, with $\operatorname{dim} A_{0}=2$, which can have a non-trivial Hopf superalgebra structure are (3|2), (11|2) and (12|2).

Proposition 4.9. Every non-trivial 4-dimensional Hopf superalgebra where $\operatorname{dim} A_{0}=2$ is isomorphic to one of the following pairwise non-isomorphic Hopf superalgebras:

1) $\mathcal{H}_{1}=\mathbb{K}\langle x, y\rangle /\left(x^{2}-x, y^{2}, y x\right)$ with $\operatorname{deg}(x)=0, \operatorname{deg}(y)=1$ and such that

$$
\begin{array}{ll}
\Delta(x)=1 \otimes x+x \otimes 1-2 x \otimes x, & \Delta(y)=1 \otimes y+y \otimes 1, \\
\varepsilon(x)=\varepsilon(y)=0, \quad S(x)=x, & S(y)=-y ;
\end{array}
$$


2) $\mathcal{H}_{2}=\mathbb{K}\langle x, y\rangle /\left(x^{2}-x, y^{2}, x y-y x-y\right)$ with $\operatorname{deg}(x)=0, \operatorname{deg}(y)=1$ and such that

$$
\begin{array}{ll}
\Delta(x)=1 \otimes x+x \otimes 1-2 x \otimes x, & \Delta(y)=1 \otimes y+y \otimes 1-2 x \otimes x y-2 x y \otimes x, \\
\varepsilon(x)=\varepsilon(y)=0, \quad S(x)=x, & S(y)=y
\end{array}
$$

3) $\mathcal{H}_{3}=\mathbb{K}\langle x, y\rangle /\left(x^{2}, y^{2}, x y+y x\right)$ with $\operatorname{deg}(x)=\operatorname{deg}(y)=1$ and such that

$$
\begin{aligned}
& \Delta(x)=1 \otimes x+x \otimes 1, \quad \Delta(y)=1 \otimes y+y \otimes 1, \\
& \varepsilon(x)=\varepsilon(y)=0, \quad S(x)=-x, \quad S(y)=-y
\end{aligned}
$$

4) $\mathcal{H}_{4}=\mathbb{K}\langle x, y\rangle /\left(x^{2}-x, y^{2}, y x\right)$ with $\operatorname{deg}(x)=0, \operatorname{deg}(y)=1$ and such that

$$
\begin{array}{ll}
\Delta(x)=1 \otimes x+x \otimes 1-2 x \otimes x, & \Delta(y)=1 \otimes y+y \otimes 1-2 x \otimes y, \\
\varepsilon(x)=\varepsilon(y)=0, \quad S(x)=x, & S(y)=2 x y-y,
\end{array}
$$

where $\mathbb{K}\langle x, y\rangle$ stands for noncommutative polynomials.

Proof. The Hopf superalgebra $\mathcal{H}_{1}$ corresponds to $A_{3 \mid 2}^{2}$ (see Appendix A.2) with $x=e_{2}^{0}, y=$ $e_{1}^{1}+e_{2}^{1}$.

The Hopf superalgebra $\mathcal{H}_{2}$ corresponds to $A_{11 \mid 2}^{1}$ with $x=e_{2}^{0}, y=e_{1}^{1}-e_{2}^{1}$.

The Hopf superalgebra $\mathcal{H}_{3}$ corresponds to $A_{12 \mid 2}^{1}$ with $x=e_{1}^{1}, y=e_{2}^{1}$.

The Hopf superalgebra $\mathcal{H}_{4}$ corresponds to $A_{3 \mid 2}^{1}$ with $x=e_{2}^{0}, y=e_{1}^{1}+e_{2}^{1}$.

Therefore, gathering the results corresponding to the case $\operatorname{dim} A_{0}=3$ and $\operatorname{dim} A_{0}=2$ we obtain five 4-dimensional Hopf superalgebras.

Theorem 4.10. Every non-trivial 4-dimensional Hopf superalgebra is isomorphic to one of the following Hopf superalgebras

$$
\begin{aligned}
& \mathcal{H}_{1} \cong \mathbb{K}[\mathbb{Z} / 2 \mathbb{Z}] \otimes \Lambda \mathbb{K}, \quad \mathcal{H}_{2} \cong \mathbb{K}[\mathbb{Z} / 2 \mathbb{Z}] \rtimes_{\sigma} \Lambda \mathbb{K}, \\
& \mathcal{H}_{3} \cong \Lambda \mathbb{K}^{2}, \quad \mathcal{H}_{4}=\mathcal{H}_{2}^{*} \quad \text { and } \quad \mathcal{H}_{5},
\end{aligned}
$$

where $\sigma: \mathbb{Z} / 2 \mathbb{Z} \rightarrow G L(\mathbb{K})$ is the non-trivial action of $\mathbb{Z} / 2 \mathbb{Z}$ on $\mathbb{K}, \mathcal{H}_{2}^{*}$ is the dual of $\mathcal{H}_{2}$ and $\mathcal{H}_{5}$ is defined as $\mathbb{K}[x, y] /\left(x^{2}+y^{2}-1, x y\right)(\operatorname{deg}(x)=0, \operatorname{deg}(y)=1)$ such that

$$
\begin{array}{lrl}
\Delta(x)=x \otimes x-\alpha y \otimes y, & \varepsilon(x)=1, & S(x)=x, \\
\Delta(y)=x \otimes y+y \otimes x, & \varepsilon(y)=0, & S(y)=\alpha y .
\end{array}
$$

with $\alpha^{4}=1$.

Proof. The first three Hopf superalgebras are cocommutative. We obtain the isomorphisms, thanks to Kostant's theorem. However, since $\mathcal{H}_{4}$ is commutative, its dual is cocommutative, it turns out that we have $\mathcal{H}_{4}=\mathcal{H}_{2}^{*}$. The Hopf superalgebra $\mathcal{H}_{5}$ corresponds to $A_{1 \mid 1}$ (Proposition 4.5$)$, it is not cocommutative and is the only one whose even part is 3-dimensional.

In the following table, we collect the results obtained for 4-dimensional superbialgebras and Hopf superalgebras. Notice that algebras are those classified by Gabriel, see Theorem 4.1. The superalgebras $(i \mid j)$ denote $j^{\text {th }}$ graduation of the $i^{\text {th }}$ algebra, obtained by Armour, Chen and Zhang, see Proposition 4.2 for the first case ( $\operatorname{dim} A_{0}=3$ ) and Proposition 4.6 for the second case $\left(\operatorname{dim} A_{0}=2\right)$. We quote below the number of corresponding superbialgebras and Hopf superalgebras. 
S. Aissaoui and A. Makhlouf

\begin{tabular}{|c|c|c|c|}
\hline algebra & superalgebra & $\sharp$ superbialgebras & $\sharp$ Hopf superalgebras \\
\hline \multirow[t]{2}{*}{1} & $1 \mid 1$ & 12 & 1 \\
\hline & $1 \mid 2$ & 0 & 0 \\
\hline \multirow[t]{3}{*}{2} & $2 \mid 1$ & 22 & 0 \\
\hline & $2 \mid 2$ & 0 & 0 \\
\hline & $2 \mid 3$ & 4 & 0 \\
\hline \multirow[t]{3}{*}{3} & $\begin{array}{ll}3 \mid 1 \\
3 \mid 1\end{array}$ & 0 & 0 \\
\hline & $3 \mid 2$ & 9 & 2 \\
\hline & $3 \mid 3$ & 0 & 0 \\
\hline 4 & $4 \mid 1$ & 3 & 0 \\
\hline 5 & $5 \mid 1$ & 0 & 0 \\
\hline \multirow[t]{2}{*}{6} & $6 \mid 1$ & 18 & 0 \\
\hline & $6 \mid 2$ & 11 & 0 \\
\hline \multirow[t]{3}{*}{7} & $7 \mid 1$ & 0 & 0 \\
\hline & $7 \mid 2$ & 0 & 0 \\
\hline & $7 \mid 3$ & 0 & 0 \\
\hline \multirow[t]{3}{*}{8} & $8 \mid 1$ & 0 & 0 \\
\hline & $8 \mid 2$ & 0 & 0 \\
\hline & $8 \mid 3$ & 0 & 0 \\
\hline \multirow[t]{2}{*}{9} & $9 \mid 1$ & 0 & 0 \\
\hline & $9 \mid 2$ & 0 & 0 \\
\hline 10 & $10 \mid 1$ & 0 & 0 \\
\hline \multirow[t]{3}{*}{11} & $11 \mid 1$ & 0 & 0 \\
\hline & $11 \mid 2$ & 1 & 1 \\
\hline & $11 \mid 3$ & 0 & 0 \\
\hline \multirow[t]{2}{*}{12} & $12 \mid 1$ & 0 & 0 \\
\hline & $12 \mid 2$ & 1 & 1 \\
\hline 13 & $13 \mid 1$ & 21 & 0 \\
\hline \multirow[t]{3}{*}{14} & 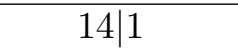 & 9 & 0 \\
\hline & $14 \mid 2$ & 4 & 0 \\
\hline & $14 \mid 3$ & 7 & 0 \\
\hline \multirow[t]{3}{*}{15} & $15 \mid 1$ & 9 & 0 \\
\hline & $15 \mid 2$ & 4 & 0 \\
\hline & $15 \mid 3$ & 7 & 0 \\
\hline \multirow[t]{3}{*}{16} & $16 \mid 2$ & 0 & 0 \\
\hline & $16 \mid 1$ & 0 & 0 \\
\hline & $16 \mid 3$ & 0 & 0 \\
\hline \multirow[t]{2}{*}{17} & $17 \mid 1$ & 11 & 0 \\
\hline & $17 \mid 2$ & 2 & 0 \\
\hline \multirow[t]{2}{*}{$(18 ; \lambda)$} & $(18 ; \lambda) \mid 1$ & 0 & 0 \\
\hline & $(18 ; \lambda) \mid 2$ & 0 & 0 \\
\hline 19 & $19 \mid 1$ & 0 & 0 \\
\hline
\end{tabular}

\section{A Appendix}

We list in the following supercoalgebras associated to a given 4-dimensional superalgebra $A$, such that $A=A_{0} \oplus A_{1}$. We denote the comultiplication by $\Delta_{i \mid j}^{k}$ and the counit by $\varepsilon_{i \mid j}^{k}$, where $i$ indicates the item of the 4-dimensional algebra listed in Theorem 4.1, and $A_{i \mid j}$ denotes the superalgebra obtained from the $i^{\text {th }}$ algebra, see Proposition 4.2 for the first case $\left(\operatorname{dim} A_{0}=3\right)$ 
and Proposition 4.6 for the second case $\left(\operatorname{dim} A_{0}=2\right)$. The exponent $k$ indicates the item of the comultiplication and counit which combined with the multiplication of superalgebra $A_{i \mid j}$ and the unit $\eta$ provide a 4-dimensional superbialgebra. Recall that for all of them we have $\eta(1)=1$ (unit element), $\Delta(1)=1 \otimes 1, \varepsilon(1)=1$ and $\varepsilon\left(A_{1}\right)=0$. In the sequel, we denote by $A_{i \mid j}^{k}$ the superbialgebra $\left(A, \mu_{i \mid j}, \eta, \Delta_{i \mid j}^{k}, \varepsilon_{i \mid j}^{k}\right)$. In order to simplify the superbialgebra in this appendix, we change the variables for superalgebras $A_{1 \mid 1}, A_{2 \mid 1}, A_{3 \mid 2}, A_{6 \mid 1}, A_{13 \mid 1}, A_{11 \mid 2}, A_{12 \mid 2}$. For the superalgebras $A_{1 \mid 1}, A_{3 \mid 2}, A_{11 \mid 2}, A_{12 \mid 2}$ we change the variables as mentioned before Proposition 4.5 and in the proof of Proposition 4.9. For $A_{2 \mid 1}$ and $A_{13 \mid 1}$ we use $x=e_{2}^{0}-e_{3}^{0}$, $y=e_{1}^{1}$, and for $A_{6 \mid 1}$ we use $x=e_{2}^{0}+e_{3}^{0}, y=e_{1}^{1}$. According to the multiplication of each superalgebra we obtain that $A_{2 \mid 1} \cong \mathbb{K}[x, y] /\left(x^{3}-x, x y, y^{2}\right), A_{6 \mid 1} \cong \mathbb{K}[x, y] /\left(x^{3}-x^{2}, x y, y^{2}\right)$, $A_{13 \mid 1} \cong \mathbb{K}\langle x, y\rangle /\left(x^{3}-x, x y+y x, x y-y, y^{2}\right)$. For the remaining superalgebras we change just the notation of basis vectors. For $\operatorname{dim}\left(A_{0}\right)=3$, we consider the basis $\left\{1=e_{1}^{0}, x=e_{2}^{0}, y=e_{3}^{0}, z=e_{1}^{1}\right\}$ and for $\operatorname{dim}\left(A_{0}\right)=2$ the basis $\left\{1=e_{1}^{0}, x=e_{2}^{0}, y=e_{1}^{1}, z=e_{2}^{1}\right\}$.

\section{A.1 Case $\operatorname{dim} A_{0}=3$}

Superalgebra $A_{1 \mid 1} \cong \mathbb{K}[x, y] /\left(x^{2}+y^{2}-1, x y\right)$ with $\operatorname{deg}(x)=0$ and $\operatorname{deg}(y)=1$, we have

1) $A_{1 \mid 1}^{1}$ with $\Delta_{1 \mid 1}^{1}(x)=\frac{1}{2}\left(x \otimes 1+x^{2} \otimes 1+x^{2} \otimes x-x \otimes x\right)$, $\Delta_{1 \mid 1}^{1}(y)=y \otimes 1+\frac{1}{2} x^{2} \otimes y-\frac{1}{2} x \otimes y, \varepsilon_{1 \mid 1}^{1}(x)=-1 ;$

2) $A_{1 \mid 1}^{2}$ with $\Delta_{1 \mid 1}^{2}(x)=x \otimes x-\alpha y \otimes y, \Delta_{1 \mid 1}^{2}(y)=x \otimes y+y \otimes x, \varepsilon_{1 \mid 1}^{2}(x)=1$, where $\alpha$ is a primitive 4 th root of unity of $\mathbb{K}$;

3) $A_{1 \mid 1}^{3}$ with $\Delta_{1 \mid 1}^{3}(x)=x \otimes x, \Delta_{1 \mid 1}^{3}(y)=1 \otimes y+y \otimes x, \varepsilon_{1 \mid 1}^{3}(x)=1$;

4) $A_{1 \mid 1}^{4}$ with $\Delta_{1 \mid 1}^{4}(x)=\frac{1}{2}\left(x^{2} \otimes x^{2}+x \otimes x^{2}+x^{2} \otimes x-x \otimes x\right)$, $\Delta_{1 \mid 1}^{4}(y)=1 \otimes y+y \otimes x^{2}, \varepsilon_{1 \mid 1}^{4}(x)=-1 ;$

5) $A_{1 \mid 1}^{5}$ with $\Delta_{1 \mid 1}^{5}(x)=\frac{1}{2}\left(x \otimes 1+1 \otimes x-1 \otimes x^{2}-x^{2} \otimes 1+x^{2} \otimes x^{2}+x \otimes x\right)$, $\Delta_{1 \mid 1}^{5}(y)=y \otimes 1+\frac{1}{2}\left(y \otimes x+x \otimes y-y \otimes x^{2}+x^{2} \otimes y\right), \varepsilon_{1 \mid 1}^{5}(x)=1 ;$

6) $A_{1 \mid 1}^{6}$ with $\Delta_{1 \mid 1}^{6}(x)=\frac{1}{2}\left(x \otimes x+x \otimes x^{2}+x^{2} \otimes x-x^{2} \otimes x^{2}\right)$, $\Delta_{1 \mid 1}^{6}(y)=1 \otimes y+y \otimes x^{2}, \varepsilon_{1 \mid 1}^{6}(x)=1 ;$

7) $A_{1 \mid 1}^{7}$ with $\Delta_{1 \mid 1}^{7}(x)=-1 \otimes 1+\frac{1}{2}\left(x \otimes x+x \otimes 1+1 \otimes x+1 \otimes x^{2}+x^{2} \otimes 1-x^{2} \otimes x^{2}\right)$, $\Delta_{1 \mid 1}^{7}(y)=\frac{1}{2}\left(x \otimes y+y \otimes x+x^{2} \otimes y+y \otimes x^{2}\right), \varepsilon_{1 \mid 1}^{7}(x)=1 ;$

8) $A_{1 \mid 1}^{8}$ with $\Delta_{1 \mid 1}^{8}(x)=x \otimes x, \Delta_{1 \mid 1}^{8}(y)=y \otimes 1+x^{2} \otimes y, \varepsilon_{1 \mid 1}^{8}(x)=1$;

9) $A_{1 \mid 1}^{9}=\left(A_{1 \mid 1}^{4}\right)^{\mathrm{cop}}, A_{1 \mid 1}^{10}=\left(A_{1 \mid 1}^{1}\right)^{\mathrm{cop}}, A_{1 \mid 1}^{11}=\left(A_{1 \mid 1}^{3}\right)^{\mathrm{cop}}, A_{1 \mid 1}^{12}=\left(A_{1 \mid 1}^{5}\right)^{\mathrm{cop}}$.

Superalgebra $A_{2 \mid 1} \cong \mathbb{K}[x, y] /\left(x^{3}-x, y^{2}, x y\right)$ with $\operatorname{deg}(x)=0$ and $\operatorname{deg}(y)=1, \varepsilon_{2 \mid 1}^{k}(x)=1$ for $k=1, \ldots, 22$ :

1) $A_{2 \mid 1}^{k}$ with $\Delta_{2 \mid 1}^{k}(x)=x \otimes x$ for $k=1, \ldots, 5$ :

- $\Delta_{2 \mid 1}^{1}(y)=y \otimes x+x \otimes y$,

- $\Delta_{2 \mid 1}^{2}(y)=x \otimes y+y \otimes x^{2}$,

- $\Delta_{2 \mid 1}^{3}(y)=y \otimes x^{2}+x^{2} \otimes y$,

- $\Delta_{2 \mid 1}^{4}(y)=1 \otimes y+y \otimes x$

- $\Delta_{2 \mid 1}^{5}(y)=1 \otimes y+y \otimes x^{2}$;

2) $A_{2 \mid 1}^{6}$ with $\Delta_{2 \mid 1}^{6}(x)=\frac{1}{2}\left(x \otimes 1+x \otimes x+x^{2} \otimes x-x^{2} \otimes 1\right), \Delta_{2 \mid 1}^{6}(y)=\frac{1}{2}\left(y \otimes x+x \otimes y+x^{2} \otimes y+y \otimes x^{2}\right)$; 
3) $A_{2 \mid 1}^{7}$ with $\Delta_{2 \mid 1}^{7}(x)=\frac{1}{4}\left(3 x \otimes x+x \otimes x^{2}+x^{2} \otimes x-x^{2} \otimes x^{2}\right)$,

$\Delta_{2 \mid 1}^{7}(y)=\frac{1}{2} y \otimes x+\frac{1}{2} x \otimes y+\frac{1}{2} x^{2} \otimes y+\frac{1}{2} y \otimes x^{2} ;$

4) $A_{2 \mid 1}^{8}$ with $\Delta_{2 \mid 1}^{8}(x)=\frac{1}{4}\left(3 x \otimes x+x \otimes x^{2}+x^{2} \otimes x-x^{2} \otimes x^{2}\right)$,

$\Delta_{2 \mid 1}^{8}(y)=1 \otimes y+\frac{1}{2} y \otimes x+\frac{1}{2} y \otimes x^{2} ;$

5) $A_{2 \mid 1}^{9}$ with $\Delta_{2 \mid 1}^{9}(x)=\frac{1}{2}\left(\frac{3}{2} x^{2} \otimes x^{2}-\frac{1}{2} x^{2} \otimes x-\frac{1}{2} x \otimes x^{2}+\frac{3}{2} x \otimes x+1 \otimes x-x \otimes 1-x^{2} \otimes 1-1 \otimes x^{2}\right)$, $\Delta_{2 \mid 1}^{9}(y)=\frac{1}{2}\left(y \otimes x+x \otimes y+x^{2} \otimes y+y \otimes x^{2}\right) ;$

6) $A_{2 \mid 1}^{10}$ with $\Delta_{2 \mid 1}^{10}(x)=\frac{1}{2}\left(x^{2} \otimes x^{2}+x \otimes x+1 \otimes x+x \otimes 1-1 \otimes x^{2}-x^{2} \otimes 1\right)$,

$\Delta_{2 \mid 1}^{10}(y)=\frac{1}{2}\left(y \otimes x+x \otimes y+x^{2} \otimes y+y \otimes x^{2}\right) ;$

7) $A_{2 \mid 1}^{11}$ with $\Delta_{2 \mid 1}^{11}(x)=\frac{1}{2}\left(x^{2} \otimes x^{2}+x \otimes x+1 \otimes x+x \otimes 1-1 \otimes x^{2}-x^{2} \otimes 1\right)$,

$\Delta_{(2 \mid 1)}^{11}(y)=1 \otimes y+\frac{1}{2}\left(y \otimes x+x \otimes y+y \otimes x^{2}-x^{2} \otimes y\right) ;$

8) $A_{2 \mid 1}^{12}$ with $\Delta_{2 \mid 1}^{12}(x)=\frac{1}{2}\left(x \otimes x^{2}+x \otimes x+1 \otimes x-1 \otimes x^{2}\right)$,

$\Delta_{2 \mid 1}^{12}(y)=1 \otimes y+\frac{1}{2}\left(y \otimes x^{2}+y \otimes x\right) ;$

9) $A_{2 \mid 1}^{13}$ with $\Delta_{2 \mid 1}^{13}(x)=-1 \otimes 1+\frac{1}{2}\left(x \otimes x+x \otimes 1+1 \otimes x+1 \otimes x^{2}+x^{2} \otimes 1-x^{2} \otimes x^{2}\right)$, $\Delta_{2 \mid 1}^{13}(y)=\frac{1}{2}\left(y \otimes x+x \otimes y+x^{2} \otimes y+y \otimes x^{2}\right) ;$

10) $A_{2 \mid 1}^{14}$ with $\Delta_{2 \mid 1}^{14}(x)=1 \otimes x+x \otimes 1-x \otimes x-x^{2} \otimes x-x \otimes x^{2}$, $\Delta_{2 \mid 1}^{14}(y)=y \otimes 1+1 \otimes y-x^{2} \otimes y-y \otimes x^{2} ;$

11) $A_{2 \mid 1}^{15}$ with $\Delta_{2 \mid 1}^{15}(x)=1 \otimes x+x \otimes 1-x^{2} \otimes x, \Delta_{2 \mid 1}^{15}(y)=y \otimes 1+1 \otimes y-x^{2} \otimes y-y \otimes x^{2}$;

12) $A_{2 \mid 1}^{16}$ with $\Delta_{2 \mid 1}^{16}(x)=1 \otimes x+x \otimes 1+\frac{1}{2}\left(-x \otimes x-x \otimes x^{2}-x^{2} \otimes x+x^{2} \otimes x^{2}\right)$, $\Delta_{2 \mid 1}^{16}(y)=y \otimes 1+1 \otimes y-x^{2} \otimes y-y \otimes x^{2}$

13) $A_{2 \mid 1}^{17}$ with $\Delta_{2 \mid 1}^{17}(x)=1 \otimes x+x \otimes 1-x \otimes x^{2}-x^{2} \otimes x+x^{2} \otimes x^{2}$, $\Delta_{2 \mid 1}^{17}(y)=y \otimes 1+1 \otimes y-x^{2} \otimes y-y \otimes x^{2} ;$

14) $A_{2 \mid 1}^{18}$ with $\Delta_{2 \mid 1}^{18}(x)=-1 \otimes 1+1 \otimes x^{2}+x^{2} \otimes 1+\frac{1}{2}\left(x \otimes x+x \otimes x^{2}+x^{2} \otimes x-3 x^{2} \otimes x^{2}\right)$, $\Delta_{2 \mid 1}^{18}(y)=\frac{1}{2} y \otimes x+\frac{1}{2} x \otimes y+\frac{1}{2} x^{2} \otimes y+\frac{1}{2} y \otimes x^{2} ;$

15) $A_{2 \mid 1}^{19}$ with $\Delta_{2 \mid 1}^{19}(x)=\frac{1}{2}\left(x \otimes x+x \otimes x^{2}+x^{2} \otimes x-x^{2} \otimes x^{2}\right), \Delta_{2 \mid 1}^{19}(y)=1 \otimes y+y \otimes x^{2}$;

16) $A_{2 \mid 1}^{20}=\left(A_{2 \mid 1}^{15}\right)^{\mathrm{cop}}, A_{2 \mid 1}^{21}=\left(A_{2 \mid 1}^{2}\right)^{\mathrm{cop}}, A_{2 \mid 1}^{22}=\left(A_{2 \mid 1}^{6}\right)^{\mathrm{cop}}$.

Superalgebra $A_{4 \mid 1}$, we have $\Delta_{4 \mid 1}^{k}(x)=x \otimes x, \varepsilon_{4 \mid 1}^{k}(x)=1, \varepsilon_{4 \mid 1}^{k}(y)=0$ for $k=1, \ldots, 3$ :

1) $A_{4 \mid 1}^{1}$ with $\Delta_{4 \mid 1}^{1}(y)=x \otimes y+y \otimes x, \Delta_{4 \mid 1}^{1}(z)=x \otimes z+z \otimes x$

2) $A_{4 \mid 1}^{2}$ with $\Delta_{4 \mid 1}^{2}(y)=x \otimes y+y \otimes 1, \Delta_{4 \mid 1}^{2}(z)=z \otimes 1+x \otimes z ;$

3) $A_{4 \mid 1}^{3}=\left(A_{4 \mid 1}^{2}\right)^{\text {cop }}$.

Superalgebra $A_{6 \mid 1} \cong \mathbb{K}[x, y] /\left(x^{3}-x^{2}, y^{2}-x y\right)$ with $\operatorname{deg}(x)=0$ and $\operatorname{deg}(y)=1$, we have $\varepsilon_{6 \mid 1}^{k}(x)=1$ for $k=1, \ldots, 18$ :

1) $A_{6 \mid 1}^{1}$ with $\Delta_{6 \mid 1}^{1}(x)=x \otimes x^{2}+x^{2} \otimes x-x^{2} \otimes x^{2}, \Delta_{6 \mid 1}^{1}(y)=y \otimes x^{2}+x^{2} \otimes y$;

2) $A_{6 \mid 1}^{2}$ with $\Delta_{6 \mid 1}^{2}(x)=x \otimes x^{2}+x^{2} \otimes x-x^{2} \otimes x^{2}+y \otimes y, \Delta_{6 \mid 1}^{2}(y)=y \otimes x^{2}+x^{2} \otimes y$;

3) $A_{6 \mid 1}^{k}$ with $\Delta_{6 \mid 1}^{k}(x)=x \otimes x$ for $k=3, \ldots, 8$ and

- $\Delta_{6 \mid 1}^{3}(y)=x \otimes y+y \otimes x$, 
- $\Delta_{6 \mid 1}^{4}(y)=y \otimes x^{2}+x \otimes y$,

- $\Delta_{6 \mid 1}^{5}(y)=y \otimes x^{2}+x^{2} \otimes y$,

- $\Delta_{6 \mid 1}^{6}(y)=y \otimes 1+x \otimes y$

- $\Delta_{6 \mid 1}^{7}(y)=y \otimes 1+x^{2} \otimes y$,

- $\Delta_{6 \mid 1}^{8}(y)=1 \otimes y+y \otimes 1$

4) $A_{6 \mid 1}^{9}$ with $\Delta_{6 \mid 1}^{9}(x)=x \otimes x+y \otimes y, \Delta_{6 \mid 1}^{9}(y)=x \otimes y+y \otimes x$;

5) $A_{6 \mid 1}^{10}$ with $\Delta_{6 \mid 1}^{10}(x)=x \otimes 1-x^{2} \otimes 1+x^{2} \otimes x, \Delta_{6 \mid 1}^{10}(y)=y \otimes x^{2}+x^{2} \otimes y$;

6) $A_{6 \mid 1}^{11}$ with $\Delta_{6 \mid 1}^{11}(x)=x \otimes x^{2}+x^{2} \otimes x-x^{2} \otimes x^{2}, \Delta_{6 \mid 1}^{11}(y)=y \otimes 1+x^{2} \otimes y$;

7) $A_{6 \mid 1}^{12}$ with $\Delta_{6 \mid 1}^{12}(x)=x \otimes 1-x^{2} \otimes 1+x^{2} \otimes x, \Delta_{6 \mid 1}^{12}(y)=x^{2} \otimes y+y \otimes 1$;

8) $A_{6 \mid 1}^{13}=\left(A_{6 \mid 1}^{4}\right)^{\mathrm{cop}}, A_{6 \mid 1}^{14}=\left(A_{6 \mid 1}^{11}\right)^{\mathrm{cop}}, A_{6 \mid 1}^{15}=\left(A_{6 \mid 1}^{6}\right)^{\mathrm{cop}}, A_{6 \mid 1}^{16}=\left(A_{6 \mid 1}^{5}\right)^{\mathrm{cop}}, A_{6 \mid 1}^{17}=\left(A_{6 \mid 1}^{12}\right)^{\mathrm{cop}}$, $A_{6 \mid 1}^{18}=\left(A_{6 \mid 1}^{10}\right)^{\mathrm{cop}}$.

Superalgebra $A_{13 \mid 1} \cong \mathbb{K}\langle x, y\rangle /\left(x^{3}-x, y^{2}, x y+y x, x y-y\right)$ with $\operatorname{deg}(x)=0$ and $\operatorname{deg}(y)=1$, we have $\varepsilon_{13 \mid 1}^{k}(x)=0$ for $k=1, \ldots, 11$ and $\varepsilon_{13 \mid 1}^{k}(x)=1$ for $k=12, \ldots, 21$ :

1) $A_{13 \mid 1}^{1}$ with $\Delta_{13 \mid 1}^{1}(x)=1 \otimes x+x \otimes 1-x^{2} \otimes x, \Delta_{13 \mid 1}^{1}(y)=1 \otimes y+y \otimes 1-x^{2} \otimes y-y \otimes x^{2}$;

2) $A_{13 \mid 1}^{2}$ with $\Delta_{13 \mid 1}^{2}(x)=1 \otimes x+x \otimes 1+\frac{1}{2} x \otimes x-\frac{1}{2} x \otimes x^{2}-\frac{1}{2} x^{2} \otimes x-\frac{1}{2} x^{2} \otimes x^{2}$, $\Delta_{13 \mid 1}^{2}(y)=1 \otimes y+y \otimes 1-x^{2} \otimes y-y \otimes x^{2} ;$

3) $A_{13 \mid 1}^{3}$ with $\Delta_{13 \mid 1}^{3}(x)=1 \otimes x+x \otimes 1+\frac{1}{2} x \otimes x-\frac{1}{2} x \otimes x^{2}-\frac{1}{2} x^{2} \otimes x-\frac{1}{2} x^{2} \otimes x^{2}$, $\Delta_{13 \mid 1}^{3}(y)=1 \otimes y+y \otimes 1 ;$

4) $A_{13 \mid 1}^{4}$ with $\Delta_{13 \mid 1}^{4}(x)=1 \otimes x+x \otimes 1+-x^{2} \otimes x-x^{2} \otimes x^{2}$, $\left.\Delta_{13 \mid 1}^{4} y\right)=1 \otimes y+y \otimes 1-y \otimes x^{2}-x^{2} \otimes y ;$

5) $A_{13 \mid 1}^{5}$ with $\Delta_{13 \mid 1}^{5}(x)=1 \otimes x+x \otimes 1-\frac{1}{2} x \otimes x-\frac{1}{2} x \otimes x^{2}-\frac{1}{2} x^{2} \otimes x+\frac{1}{2} x^{2} \otimes x^{2}$, $\Delta_{13 \mid 1}^{5}(y)=1 \otimes y+y \otimes 1-\frac{1}{2} x \otimes y-\frac{1}{2} y \otimes x-\frac{1}{2} x^{2} \otimes y-\frac{1}{2} y \otimes x^{2} ;$

6) $A_{13 \mid 1}^{6}$ with $\Delta_{13 \mid 1}^{6}(x)=1 \otimes x+x \otimes 1-\frac{1}{2} x \otimes x-\frac{1}{2} x \otimes x^{2}-\frac{1}{2} x^{2} \otimes x+\frac{1}{2} x^{2} \otimes x^{2}-2 y \otimes y$, $\Delta_{13 \mid 1}^{6}(y)=1 \otimes y+y \otimes 1-\frac{1}{2} x \otimes y-\frac{1}{2} y \otimes x-\frac{1}{2} x^{2} \otimes y-\frac{1}{2} y \otimes x^{2} ;$

7) $A_{13 \mid 1}^{7}$ with $\Delta_{13 \mid 1}^{7}(x)=1 \otimes x+x \otimes 1-\frac{1}{2} x \otimes x-\frac{1}{2} x \otimes x^{2}+\frac{1}{2} x^{2} \otimes x^{2}-x^{2} \otimes x$, $\Delta_{13 \mid 1}^{7}(y)=1 \otimes y+y \otimes 1-\frac{1}{2} x \otimes y-\frac{1}{2} x^{2} \otimes y-y \otimes x^{2} ;$

8) $A_{13 \mid 1}^{8}$ with $\Delta_{13 \mid 1}^{8}(x)=1 \otimes x+x \otimes 1-x \otimes x^{2}, \Delta_{13 \mid 1}^{8}(y)=1 \otimes y+y \otimes 1-x^{2} \otimes y-y \otimes x^{2}$;

9) $A_{13 \mid 1}^{9}$ with $\Delta_{13 \mid 1}^{9}(x)=1 \otimes x+x \otimes 1-x \otimes x^{2}, \Delta_{13 \mid 1}^{9}(y)=y \otimes 1+1 \otimes y-y \otimes x^{2}$;

10) $A_{13 \mid 1}^{10}=\left(A_{13 \mid 1}^{9}\right)^{\text {cop }}$

11) $A_{13 \mid 1}^{11}$ with $\Delta_{13 \mid 1}^{11}(x)=1 \otimes x+x \otimes 1-x \otimes x-x \otimes x^{2}-x^{2} \otimes x$, $\Delta_{13 \mid 1}^{11}(y)=1 \otimes y+y \otimes 1-x^{2} \otimes y-y \otimes x^{2} ;$

12) $A_{13 \mid 1}^{12}$ with $\Delta_{13 \mid 1}^{12}(x)=\frac{1}{2} 1 \otimes x+\frac{1}{2} x \otimes 1-\frac{1}{2} 1 \otimes x^{2}-\frac{1}{2} x^{2} \otimes 1-\frac{1}{4} x \otimes x^{2}-\frac{1}{4} x^{2} \otimes x+\frac{3}{4} x \otimes x+\frac{3}{4} x^{2} \otimes x^{2}$, $\Delta_{13 \mid 1}^{12}(y)=\frac{1}{2} x \otimes y+\frac{1}{2} y \otimes x+\frac{1}{2} x^{2} \otimes y+\frac{1}{2} y \otimes x^{2} ;$

13) $A_{13 \mid 1}^{k}$ with $\Delta_{13 \mid 1}^{k}(y)=\frac{1}{2} x \otimes y+\frac{1}{2} y \otimes x+\frac{1}{2} x^{2} \otimes y+\frac{1}{2} y \otimes x^{2}$ for $k=13, \ldots, 21$ :

- $\Delta_{13 \mid 1}^{13}(x)=x \otimes x$,

- $\Delta_{13 \mid 1}^{14}(x)=\frac{1}{2} x \otimes x+\frac{1}{2} x \otimes x^{2}+\frac{1}{2} x^{2} \otimes x-\frac{1}{2} x^{2} \otimes x^{2}+2 y \otimes y$, 
- $\Delta_{13 \mid 1}^{15}(x)=\frac{1}{2} x \otimes x+\frac{1}{2} x^{2} \otimes x+\frac{1}{2} x \otimes 1-\frac{1}{2} x^{2} \otimes 1$,

- $\Delta_{13 \mid 1}^{16}(x)=\frac{1}{2} 1 \otimes x+\frac{1}{2} x \otimes 1-\frac{1}{2} 1 \otimes x^{2}-\frac{1}{2} x^{2} \otimes 1+\frac{1}{2} x \otimes x+\frac{1}{2} x^{2} \otimes x^{2}$,

- $\Delta_{13 \mid 1}^{17}(x)=\frac{3}{4} x \otimes x+\frac{1}{4} x \otimes x^{2}+\frac{1}{4} x^{2} \otimes x-\frac{1}{4} x^{2} \otimes x^{2}$,

- $\Delta_{13 \mid 1}^{18}(x)=-1 \otimes 1+\frac{1}{2} x \otimes x+1 \otimes x^{2}+x^{2} \otimes 1+\frac{1}{2} x \otimes x^{2}+\frac{1}{2} x^{2} \otimes x-\frac{3}{2} x^{2} \otimes x^{2}$,

- $\Delta_{13 \mid 1}^{19}(x)=-1 \otimes 1+\frac{1}{2} x \otimes x+1 \otimes x^{2}+x^{2} \otimes 1+\frac{1}{2} x \otimes x^{2}+\frac{1}{2} x^{2} \otimes x-\frac{3}{2} x^{2} \otimes x^{2}+2 y \otimes y$,

- $\Delta_{13 \mid 1}^{20}(x)=-1 \otimes 1+\frac{1}{2} x \otimes x+\frac{1}{2} 1 \otimes x^{2}+\frac{1}{2} x^{2} \otimes 1+\frac{1}{2} x \otimes x^{2}+\frac{1}{2} x^{2} \otimes x-\frac{3}{2} x^{2} \otimes x^{2}$;

14) $A_{13 \mid 1}^{21}=\left(A_{13 \mid 1}^{15}\right)^{\mathrm{cop}}$.

Superalgebra $A_{14 \mid 1}$, we have $\varepsilon_{14 \mid 1}^{k}(x)=0, \varepsilon_{14 \mid 1}^{k}(y)=0$ for $k=1, \ldots, 9$ :

1) $A_{14 \mid 1}^{k}$ with $\Delta_{14 \mid 1}^{1}(x)=1 \otimes x+x \otimes 1-x \otimes x$ for $k=1, \ldots, 6$ :

- $\Delta_{14 \mid 1}^{1}(y)=1 \otimes y+y \otimes 1-x \otimes y-y \otimes x, \Delta_{14 \mid 1}^{1}(z)=1 \otimes z+z \otimes 1$,

- $\Delta_{14 \mid 1}^{2}(y)=1 \otimes y+y \otimes 1-x \otimes y-y \otimes x, \Delta_{14 \mid 1}^{2}(z)=1 \otimes z+z \otimes 1-x \otimes z$,

- $\Delta_{14 \mid 1}^{3}(y)=1 \otimes y+y \otimes 1-x \otimes y-y \otimes x+y \otimes y, \Delta_{14 \mid 1}^{3}(z)=1 \otimes z+z \otimes 1-x \otimes z$,

- $\Delta_{14 \mid 1}^{4}(y)=1 \otimes y+y \otimes 1-x \otimes y-y \otimes x, \Delta_{14 \mid 1}^{4}(z)=1 \otimes z+z \otimes 1-x \otimes z-z \otimes x$,

- $\Delta_{14 \mid 1}^{5}(y)=1 \otimes y+y \otimes 1-x \otimes y-y \otimes x+y \otimes y, \Delta_{14 \mid 1}^{5}(z)=1 \otimes z+z \otimes 1-x \otimes z-z \otimes x+z \otimes y$,

- $\Delta_{14 \mid 1}^{6}(y)=1 \otimes y+y \otimes 1-x \otimes z-z \otimes x+y \otimes y, \Delta_{14 \mid 1}^{6}(z)=1 \otimes z+z \otimes 1-x \otimes z-z \otimes x ;$

2) $A_{14 \mid 1}^{7}$ with $\Delta_{14 \mid 1}^{7}(x)=1 \otimes x+x \otimes 1-x \otimes x+y \otimes y$,

$\Delta_{14 \mid 1}^{7}(y)=1 \otimes y+y \otimes 1-x \otimes y-y \otimes x+y \otimes y, \Delta_{14 \mid 1}^{7}(z)=1 \otimes z+z \otimes 1 ;$

3) $A_{14 \mid 1}^{8}=\left(A_{14 \mid 1}^{2}\right)^{\mathrm{cop}}, A_{14 \mid 1}^{9}=\left(A_{14 \mid 1}^{3}\right)^{\mathrm{cop}}$.

Superalgebra $A_{14 \mid 2}$, we have $\varepsilon_{14 \mid 2}^{k}(x)=0$ and $\varepsilon_{14 \mid 2}^{k}(y)=0$ for $k=1, \ldots, 4$ :

1) $A_{14 \mid 2}^{1}$ with $\Delta_{14 \mid 2}^{1}(x)=1 \otimes x+x \otimes 1-x \otimes x, \Delta_{14 \mid 2}^{1}(y)=1 \otimes y+y \otimes 1-y \otimes z-z \otimes x$, $\Delta_{14 \mid 2}^{1}(z)=1 \otimes z+z \otimes 1-z \otimes x-x \otimes z ;$

2) $A_{14 \mid 2}^{2}$ with $\Delta_{14 \mid 2}^{2}(x)=1 \otimes x+x \otimes 1-x \otimes x, \Delta_{14 \mid 2}^{2}(y)=1 \otimes y+y \otimes 1-x \otimes y$, $\Delta_{14 \mid 2}^{2}(z)=1 \otimes z+z \otimes 1-x \otimes z-z \otimes x$

3) $A_{14 \mid 2}^{3}$ with $\Delta_{14 \mid 2}^{3}(x)=1 \otimes x+x \otimes 1-x \otimes x, \Delta_{14 \mid 2}^{3}(y)=1 \otimes y+y \otimes 1-x \otimes y-y \otimes x+y \otimes y$, $\Delta_{14 \mid 2}^{3}(z)=1 \otimes z+z \otimes 1-z \otimes x-x \otimes z ;$

4) $A_{14 \mid 2}^{4}=\left(A_{14 \mid 2}^{2}\right)^{\text {cop }}$.

Superalgebra $A_{15 \mid 1}$, we have $A_{15 \mid 1}^{1}=\left(A_{14 \mid 1}^{1}\right)^{\mathrm{op}}, A_{15 \mid 1}^{2}=\left(A_{14 \mid 1}^{2}\right)^{\mathrm{op}}, A_{15 \mid 1}^{3}=\left(A_{14 \mid 1}^{5}\right)^{\mathrm{op}}, A_{15 \mid 1}^{4}=$ $\left(A_{14 \mid 1}^{9}\right)^{\mathrm{op}}, A_{15 \mid 1}^{5}=\left(A_{14 \mid 1}^{6}\right)^{\mathrm{op}}, A_{15 \mid 1}^{6}=\left(A_{14 \mid 1}^{3}\right)^{\mathrm{op}}, A_{15 \mid 1}^{7}=\left(A_{14 \mid 1}^{4}\right)^{\mathrm{op}}, A_{15 \mid 1}^{8}=\left(A_{14 \mid 1}^{7}\right)^{\mathrm{op}}, A_{15 \mid 1}^{9}=$ $\left(A_{14 \mid 1}^{4}\right)^{\mathrm{op}, \mathrm{cop}}$.

Superalgebra $A_{15 \mid 2}$, we have $A_{15 \mid 2}^{1}=\left(A_{14 \mid 2}^{1}\right)^{\mathrm{op}}, A_{15 \mid 2}^{2}=\left(A_{14 \mid 2}^{4}\right)^{\mathrm{op}}, A_{15 \mid 2}^{3}=\left(A_{14 \mid 2}^{2}\right)^{\mathrm{op}}, A_{15 \mid 2}^{4}=$ $\left(A_{14 \mid 2}^{3}\right)^{\text {op }}$.

Superalgebra $A_{17 \mid 1}$, we have $\varepsilon_{17 \mid 1}^{k}(x)=1$ and $\varepsilon_{17 \mid 1}^{k}(y)=0$ for $k=1, \ldots, 11$ :

1) $A_{17 \mid 1}^{1}$ with $\Delta_{17 \mid 1}^{1}(x)=x \otimes x+y \otimes y+z \otimes z, \Delta_{17 \mid 1}^{1}(y)=x \otimes y+y \otimes x+z \otimes z$, $\Delta_{17 \mid 1}^{1}(z)=x \otimes z+z \otimes x+z \otimes y+y \otimes z ;$

2) $A_{17 \mid 1}^{2}$ with $\Delta_{17 \mid 1}^{2}(x)=x \otimes x+z \otimes z, \Delta_{17 \mid 1}^{2}(y)=x \otimes y+y \otimes x, \Delta_{17 \mid 1}^{2}(z)=z \otimes x+x \otimes z$; 
3) $A_{17 \mid 1}^{3}$ with $\Delta_{17 \mid 1}^{3}(x)=x \otimes x+z \otimes z, \Delta_{17 \mid 1}^{3}(y)=x \otimes y+y \otimes x+z \otimes z-y \otimes y$,

$\Delta_{17 \mid 1}^{3}(z)=z \otimes x+x \otimes z ;$

4) $A_{17 \mid 1}^{4}$ with $\Delta_{17 \mid 1}^{4}(x)=x \otimes x+y \otimes y, \Delta_{17 \mid 1}^{4}(y)=x \otimes y+y \otimes x$,

$\Delta_{17 \mid 1}^{4}(z)=z \otimes x+x \otimes z-z \otimes y-y \otimes z ;$

5) $A_{17 \mid 1}^{k}$, we have $\Delta_{17 \mid 1}^{k}(x)=x \otimes x$ and $\Delta_{17 \mid 1}^{k}(y)=x \otimes y+y \otimes x+y \otimes y$ for $k=5,6,7$ :

- $\Delta_{17 \mid 1}^{5}(z)=x \otimes z+z \otimes x$,

- $\Delta_{17 \mid 1}^{6}(z)=x \otimes z+z \otimes x+z \otimes y-y \otimes z$,

- $\Delta_{17 \mid 1}^{7}(z)=z \otimes x+x \otimes z+z \otimes y$;

6) $A_{17 \mid 1}^{8}=\left(A_{17 \mid 1}^{7}\right)^{\mathrm{cop}}$

7) $A_{17 \mid 1}^{k}$, we have $\Delta_{17 \mid 1}^{k}(x)=x \otimes x$ for $k=9,10,11$ :

- $\Delta_{17 \mid 1}^{9}(y)=x \otimes y+y \otimes x-y \otimes y, \Delta_{17 \mid 1}^{9}(z)=z \otimes x+x \otimes z$,

- $\Delta_{17 \mid 1}^{10}(y)=x \otimes y+y \otimes x+z \otimes z, \Delta_{17 \mid 1}^{10}(z)=x \otimes z+z \otimes x$,

- $\Delta_{17 \mid 1}^{11}(y)=x \otimes y+y \otimes x+y \otimes y+z \otimes z, \Delta_{17 \mid 1}^{11}(z)=z \otimes x+x \otimes z+z \otimes y+y \otimes z$.

\section{A.2 Case $\operatorname{dim} A_{0}=2$}

Superalgebra $A_{2 \mid 3}$, we have $\Delta_{2 \mid 3}^{k}(x)=1 \otimes x+x \otimes 1-x \otimes x, \varepsilon_{2 \mid 3}^{k}(x)=0$ for $k=1, \ldots, 4$ :

1) $A_{2 \mid 3}^{1}$ with $\Delta_{2 \mid 3}^{1}(y)=1 \otimes y+y \otimes 1-x \otimes y, \Delta_{2 \mid 3}^{1}(z)=1 \otimes z+z \otimes 1-x \otimes z-z \otimes x$;

2) $A_{2 \mid 3}^{2}$ with $\Delta_{2 \mid 3}^{2}(y)=1 \otimes y+y \otimes 1-x \otimes y+z \otimes x, \Delta_{2 \mid 3}^{2}(z)=1 \otimes z+z \otimes 1-x \otimes z-z \otimes x$;

3) $A_{2 \mid 3}^{3}=\left(A_{2 \mid 3}^{1}\right)^{\mathrm{cop}}, A_{2 \mid 3}^{4}=\left(A_{2 \mid 3}^{2}\right)^{\mathrm{cop}}$.

Superalgebra $A_{3 \mid 2} \cong \mathbb{K}\langle x, y\rangle /\left(x^{2}-x, y^{2}, y x\right)$ with $\operatorname{deg}(x)=0$ and $\operatorname{deg}(y)=1$, we have $\varepsilon_{3 \mid 2}^{k}(x)=0$ for $k=1, \ldots, 9$ :

1) $A_{3 \mid 2}^{k}$ with $\Delta_{3 \mid 2}^{k}(x)=1 \otimes x+x \otimes 1-2 x \otimes x$ for $k=1,2$, and

- $\Delta_{3 \mid 2}^{1}(y)=1 \otimes y+y \otimes 1-2 x \otimes y$,

- $\Delta_{3 \mid 2}^{2}(y)=1 \otimes y+y \otimes 1$

2) $A_{3 \mid 2}^{k}$ with $\Delta_{3 \mid 2}^{k}(x)=1 \otimes x+x \otimes 1-x \otimes x$, for $k=3, \ldots, 9$, and

- $\Delta_{3 \mid 2}^{3}(y)=1 \otimes y+y \otimes 1-x \otimes y-y \otimes x+x y \otimes x+x \otimes x y$,

- $\Delta_{3 \mid 2}^{4}(y)=1 \otimes y+y \otimes 1-x \otimes y-x y \otimes x$,

- $\Delta_{3 \mid 2}^{5}(y)=1 \otimes y+y \otimes 1-x \otimes y-y \otimes x$,

- $\Delta_{3 \mid 2}^{6}(y)=1 \otimes y+y \otimes 1-x \otimes x y-x y \otimes x$,

- $\Delta_{3 \mid 2}^{7}(y)=1 \otimes y+y \otimes 1-y \otimes x$

- $\Delta_{3 \mid 2}^{8}(y)=1 \otimes y+y \otimes 1$

- $A_{3 \mid 2}^{9}=\left(A_{3 \mid 2}^{4}\right)^{\mathrm{cop}}$.

Superalgebra $A_{6 \mid 2}$, we have $\Delta_{6 \mid 2}^{k}(x)=x \otimes x$ and $\varepsilon_{6 \mid 2}^{k}(x)=1$ for $k=1, \ldots, 11$ :

1) $A_{6 \mid 2}^{k}$ with $\Delta_{6 \mid 2}^{k}(y)=y \otimes x+x \otimes y$ for $k=1, \ldots, 4$, and 
- $\Delta_{6 \mid 2}^{1}(z)=z \otimes 1+1 \otimes z$

- $\Delta_{6 \mid 2}^{2}(z)=z \otimes x+x \otimes z$,

- $\Delta_{6 \mid 2}^{3}(z)=z \otimes x+1 \otimes z$

- $\Delta_{6 \mid 2}^{4}(z)=1 \otimes z+z \otimes x+1 \otimes y-x \otimes y$;

2) $A_{6 \mid 2}^{5}$ with $\Delta_{6 \mid 2}^{6}(y)=y \otimes 1+1 \otimes y, \Delta_{6 \mid 2}^{5}(z)=z \otimes x+x \otimes z$;

3) $A_{6 \mid 2}^{6}$ with $\Delta_{6 \mid 2}^{6}(y)=y \otimes x+1 \otimes y, \Delta_{6 \mid 2}^{6}(z)=z \otimes x+1 \otimes z$;

4) $A_{6 \mid 2}^{7}$ with $\Delta_{6 \mid 2}^{7}(y)=1 \otimes y+y \otimes x, \Delta_{6 \mid 2}^{7}(z)=x \otimes z+z \otimes x$;

5) $A_{6 \mid 2}^{8}$ with $\Delta_{6 \mid 2}^{8}(y)=y \otimes x+x \otimes y+z \otimes 1+1 \otimes z-x \otimes z-z \otimes x, \Delta_{6 \mid 2}^{8}(z)=1 \otimes z+z \otimes 1$;

6) $A_{6 \mid 2}^{9}$ with $\Delta_{6 \mid 2}^{9}(y)=y \otimes x+1 \otimes y+z \otimes 1+1 \otimes z-z \otimes x-x \otimes z, \Delta_{6 \mid 2}^{9}(z)=z \otimes x+1 \otimes z$;

7) $A_{6 \mid 2}^{10}$ with $\Delta_{6 \mid 2}^{10}(y)=y \otimes x+\frac{1}{2} x \otimes y+\frac{1}{2} 1 \otimes y+\frac{1}{4} 1 \otimes z-\frac{1}{4} x \otimes z$, $\Delta_{6 \mid 2}^{10}(z)=z \otimes x+\frac{1}{2} 1 \otimes z+1 \otimes y-x \otimes y+\frac{1}{2} x \otimes z ;$

8) $A_{6 \mid 2}^{11}=\left(A_{3 \mid 2}^{6}\right)^{\text {cop }}$.

Superalgebra $A_{11 \mid 2} \cong \mathbb{K}\langle x, y\rangle /\left(x^{2}-x, y^{2}, x y-y x-y\right)$ with $\operatorname{deg}(x)=0, \operatorname{deg}(y)=1$ :

1) $\Delta_{11 \mid 2}^{1}(x)=1 \otimes x+x \otimes 1-2 x \otimes x, \Delta_{11 \mid 2}^{1}(y)=1 \otimes y+y \otimes 1-2 x \otimes x y-2 x y \otimes x$, $\varepsilon_{11 \mid 2}^{1}(x)=1$.

Superalgebra $A_{12 \mid 2}^{1} \cong \mathbb{K}\langle x, y\rangle /\left(x^{2}, y^{2}, x y+y x\right)$ with $\operatorname{deg}(x)=\operatorname{deg}(y)=1$ :

1) $\Delta_{12 \mid 2}^{1}(x)=1 \otimes x+x \otimes 1, \Delta_{12 \mid 2}^{1}(y)=1 \otimes y+y \otimes 1$.

Superalgebra $A_{14 \mid 3}$, we have $\Delta_{14 \mid 3}^{k}(z)=1 \otimes z+z \otimes 1-x \otimes z-z \otimes x$ and $\varepsilon_{14 \mid 3}^{k}(x)=0$ for $k=1, \ldots, 7$ :

1) $A_{14 \mid 3}^{k}$ with $\Delta_{14 \mid 3}^{k}(x)=1 \otimes x+x \otimes 1-x \otimes x$ for $k=1, \ldots, 4:$

- $\Delta_{14 \mid 3}^{1}(y)=1 \otimes y+y \otimes 1$,

- $\Delta_{14 \mid 3}^{2}(y)=1 \otimes y+y \otimes 1+x \otimes z+z \otimes x$,

- $\Delta_{14 \mid 3}^{3}(y)=1 \otimes y+y \otimes 1-x \otimes y$,

- $\Delta_{14 \mid 3}^{4}(y)=1 \otimes y+y \otimes 1-x \otimes y-y \otimes x ;$

2) $A_{14 \mid 3}^{k}$ with $\Delta_{14 \mid 3}^{k}(x)=1 \otimes x+x \otimes 1-x \otimes x+z \otimes z$ for $k=5,6$ :

- $\Delta_{14 \mid 3}^{5}(y)=1 \otimes y+y \otimes 1$,

- $\Delta_{14 \mid 3}^{6}(y)=1 \otimes y+y \otimes 1+x \otimes z+z \otimes x ;$

3) $A_{14 \mid 3}^{7}=\left(A_{14 \mid 3}^{3}\right)^{\text {cop }}$.

Superalgebra $A_{15 \mid 3}$, we have $A_{15 \mid 3}^{1}=\left(A_{14 \mid 3}^{1}\right)^{\mathrm{op}}, A_{15 \mid 3}^{2}=\left(A_{14 \mid 3}^{2}\right)^{\mathrm{op}}, A_{15 \mid 3}^{3}=\left(A_{14 \mid 3}^{3}\right)^{\mathrm{op}}, A_{15 \mid 3}^{4}=$ $\left(A_{14 \mid 3}^{4}\right)^{\mathrm{op}}, A_{15 \mid 3}^{5}=\left(A_{14 \mid 3}^{5}\right)^{\mathrm{op}}, A_{15 \mid 3}^{6}=\left(A_{14 \mid 3}^{6}\right)^{\mathrm{op}}, A_{15 \mid 3}^{7}=\left(A_{14 \mid 3}^{5}\right)^{\mathrm{op}, \mathrm{cop}}$.

Superalgebra $A_{17 \mid 2}$, we have:

1) $A_{17 \mid 2}^{1}$ with $\Delta_{17 \mid 2}^{1}(x)=1 \otimes x+x \otimes 1-x \otimes x, \Delta_{17 \mid 2}^{1}(y)=1 \otimes y+y \otimes 1-y \otimes x-x \otimes y$, $\Delta_{17 \mid 2}^{1}(z)=1 \otimes z+z \otimes 1-z \otimes x-x \otimes z, \varepsilon_{15 \mid 3}^{7}(x)=0$

2) $A_{17 \mid 2}^{2}$ with $\Delta_{17 \mid 2}^{2}(x)=x \otimes x, \Delta_{17 \mid 2}^{2}(y)=x \otimes y+y \otimes x, \Delta_{17 \mid 2}^{2}(z)=x \otimes z+z \otimes x, \varepsilon_{15 \mid 3}^{7}(x)=1$. 


\section{Acknowledgements}

The authors are grateful to the referees for their valuable remarks and suggestions.

\section{References}

[1] Abe E., Hopf algebras, Cambridge Tracts in Mathematics, Vol. 74, Cambridge University Press, Cambridge, 1980.

[2] Andruskiewitsch N., About finite dimensional Hopf algebras, in Quantum Symmetries in Theoretical Physics and Mathematics (Bariloche, 2000), Contemp. Math., Vol. 294, Amer. Math. Soc., Providence, RI, 2002, $1-57$.

[3] Andruskiewitsch N., Angiono I., Yamane H., On pointed Hopf superalgebras, in New developments in Lie theory and its applications, Contemp. Math., Vol. 544, Amer. Math. Soc., Providence, RI, 2011, 123-140, arXiv:1009.5148.

[4] Andruskiewitsch N., Etingof P., Gelaki S., Triangular Hopf algebras with the Chevalley property, Michigan Math. J. 49 (2001), 277-298, math.QA/0008232.

[5] Andruskiewitsch N., Schneider H.J., On the classification of finite-dimensional pointed Hopf algebras, Ann. of Math. 171 (2010), 375-417, math.QA/0502157.

[6] Armour A., The algebraic and geometric classification of four dimensional super-algebras, Master Thesis, Victoria University of Wellington, 2006.

[7] Armour A., Chen H.X., Zhang Y., Classification of 4-dimensional graded algebras, Comm. Algebra 37 (2009), 3697-3728.

[8] Beattie M., Dăscălescu S., Hopf algebras of dimension 14, J. London Math. Soc. 69 (2004), 65-78, math.QA/0205243.

[9] Beattie M., Dăscălescu S., Grünenfelder L., On the number of types of finite-dimensional Hopf algebras, Invent. Math. 136 (1999), 1-7.

[10] Beattie M., García G.A., Classifying Hopf algebras of a given dimension, in Hopf Algebras and Tensor Categories, Contemp. Math., Vol. 585, Amer. Math. Soc., Providence, RI, 2013, 125-152, arXiv:1206.6529.

[11] Cheng Y.L., Ng S.H., On Hopf algebras of dimension 4p, J. Algebra 328 (2011), 399-419.

[12] Connes A., Kreimer D., Hopf algebras, renormalization and noncommutative geometry, Comm. Math. Phys. 199 (1998), 203-242, hep-th/9808042.

[13] Deguchi T., Fujii A., Ito K., Quantum superalgebra $U_{q} \operatorname{osp}(2,2)$, Phys. Lett. B 238 (1990), $242-246$.

[14] Dekkar K., Makhlouf A., Bialgebra structures of 2-associative algebras, Arab. J. Sci. Eng. Sect. C Theme Issues 33 (2008), 137-151.

[15] Etingof P., Gelaki S., The classification of finite-dimensional triangular Hopf algebras over an algebraically closed field of characteristic 0, Mosc. Math. J. 3 (2003), 37-43.

[16] Fukuda N., Semisimple Hopf algebras of dimension 12, Tsukuba J. Math. 21 (1997), 43-54.

[17] Gabriel P., Finite representation type is open, in Proceedings of the International Conference on Representations of Algebras (Carleton Univ., Ottawa, Ont., 1974), Lecture Notes in Math., Vol. 488, Springer, Berlin, 1975, 132-155.

[18] Gould M.D., Zhang R.B., Bracken A.J., Quantum double construction for graded Hopf algebras, Bull. Austral. Math. Soc. 47 (1993), 353-375.

[19] Guichardet A., Groupes quantiques. Introduction au point de vue formel, Savoirs Actuels, InterEditions, Paris, 1995.

[20] Holtkamp R., Comparison of Hopf algebras on trees, Arch. Math. (Basel) 80 (2003), 368-383.

[21] Kassel C., Quantum groups, Graduate Texts in Mathematics, Vol. 155, Springer-Verlag, New York, 1995.

[22] Kreimer D., On the Hopf algebra structure of perturbative quantum field theories, Adv. Theor. Math. Phys. 2 (1998), 303-334, q-alg/9707029.

[23] Kulish P.P., Reshetikhin N.Y., Universal R-matrix of the quantum superalgebra osp(2|1), Lett. Math. Phys. 18 (1989), 143-149.

[24] Majid S., Cross products by braided groups and bosonization, J. Algebra 163 (1994), 165-190. 
[25] Majid S., Foundations of quantum group theory, Cambridge University Press, Cambridge, 1995.

[26] Makhlouf A., Degeneration, rigidity and irreducible components of Hopf algebras, Algebra Colloq. 12 (2005), 241-254, math.RA/0211187.

[27] Makhlouf A., Algèbre de Hopf et renormalisation en théorie quantique des champs, in Théorie Quantique des Champs: Méthodes et Applications, Editors T. Boudjedaa, A. Makhlouf, Travaux en Cours, Hermann, Paris, 2007, 191-242.

[28] Masuoka A., Semisimple Hopf algebras of dimension 6, 8, Israel J. Math. 92 (1995), 361-373.

[29] Milnor J.W., Moore J.C., On the structure of Hopf algebras, Ann. of Math. 81 (1965), 211-264.

[30] Montgomery S., Hopf algebras and their actions on rings, CBMS Regional Conference Series in Mathematics, Vol. 82, Conference Board of the Mathematical Sciences, Washington, DC, 1993.

[31] Montgomery S., Classifying finite-dimensional semisimple Hopf algebras, in Trends in the Representation Theory of Finite-Dimensional Algebras (Seattle, WA, 1997), Contemp. Math., Vol. 229, Amer. Math. Soc., Providence, RI, 1998, 265-279.

[32] Natale S., Hopf algebras of dimension 12, Algebr. Represent. Theory 5 (2002), 445-455.

[33] Ng S.H., Non-semisimple Hopf algebras of dimension $p^{2}$, J. Algebra 255 (2002), 182-197, math.QA/0110223.

[34] Scheunert M., Zhang R.B., Integration on Lie supergroups: a Hopf superalgebra approach, J. Algebra 292 (2005), 324-342, math.RA/0012052.

[35] Shnider S., Sternberg S., Quantum groups. From coalgebras to Drinfel'd algebras. A guided tour, Graduate Texts in Mathematical Physics, Vol. 2, International Press, Cambridge, MA, 1993.

[36] Ştefan D., The set of types of $n$-dimensional semisimple and cosemisimple Hopf algebras is finite, J. Algebra 193 (1997), 571-580.

[37] Ştefan D., Hopf algebras of low dimension, J. Algebra 211 (1999), 343-361.

[38] Williams R.E., Finite dimensional Hopf algebras, Ph.D. Thesis, The Florida State University, 1988.

[39] Zhu Y., Hopf algebras of prime dimension, Int. Math. Res. Not. 1994 (1994), 53-59. 